\title{
The GMT-Consortium Large Earth Finder (G-CLEF): An optical echelle spectrograph for the Giant Magellan Telescope (GMT)
}

Andrew Szentgyorgyi ${ }^{\mathrm{a}}$, Daniel Baldwin ${ }^{\mathrm{a}}$, Stuart Barnes ${ }^{\mathrm{a}}$, Jacob Bean ${ }^{\mathrm{e}}$, Sagi Ben-Ami ${ }^{\mathrm{a}}$, Patricia Brennan $^{\mathrm{a}}$, Jamie Budynkiewicz ${ }^{\mathrm{a}}$, Moo-Yung Chun ${ }^{\mathrm{f}}$, Charlie Conroy ${ }^{\mathrm{a}}$, Jeffrey D. Crane ${ }^{\mathrm{c}}$, Harland Epps $^{\mathrm{h}}$, Ian Evans ${ }^{\mathrm{a}}$, Janet Evans ${ }^{\mathrm{a}}$, Jeff Foster ${ }^{\mathrm{a}}$, Anna Frebel ${ }^{\mathrm{g}}$, Thomas Gauron ${ }^{\mathrm{a}}$, Dani Guzman ${ }^{\mathrm{d}}$, Tyson

Hare $^{\mathrm{c}}$, Bi-Ho Jang ${ }^{\mathrm{f}}$, Jeong-Gyun Jang ${ }^{\mathrm{f}}$, Andres Jordan ${ }^{\mathrm{d}}$, Jihun Kim ${ }^{\mathrm{f}}$, Kang-Min Kim ${ }^{\mathrm{f}}$, Claudia Mendes de Oliveira ${ }^{\mathrm{i}}$, Mercedes Lopez-Morales ${ }^{\mathrm{a}}$, Kenneth McCracken ${ }^{\mathrm{a}}$, Stuart McMuldroch ${ }^{\mathrm{a}}$, Joseph Miller $^{\mathrm{a}}$, Mark Mueller ${ }^{\mathrm{a}}$, Jae Sok Oh ${ }^{\mathrm{f}}, \mathrm{Cem}$ Onyuksel ${ }^{\mathrm{a}}$, Mark Ordway ${ }^{\mathrm{a}}$, Byeong-Gon Park ${ }^{\mathrm{f}}$, Chan Park $^{\mathrm{f}}$, Sung-Joon Park ${ }^{\mathrm{f}}$, Charles Paxson ${ }^{\mathrm{a}}$, David Phillips ${ }^{\mathrm{a}}$, David Plummer ${ }^{\mathrm{a}}$, William Podgorski ${ }^{\mathrm{a}}$, Andreas Seifahrt ${ }^{\mathrm{e}}$, Daniel Stark ${ }^{\mathrm{b}}$, Joao Steiner ${ }^{\mathrm{i}}$, Alan Uomoto ${ }^{\mathrm{c}}$, Ronald Walsworth ${ }^{\mathrm{a}}$ and Young-Sam $\mathrm{Yu}^{\mathrm{f}}$.

${ }^{a}$ Harvard-Smithsonian Center for Astrophysics, 60 Garden St., Cambridge, MA 02140;

${ }^{\mathrm{b}}$ Steward Observatory, University of Arizona, 933 North Cherry St., Tucson, AZ, 85721

${ }^{\mathrm{c}}$ The Observatories of the Carnegie Institution for Science, 813 Santa Barbara St., Pasadena, CA

91101;

d Pontificia Universidad Catolica de Chile, Vicuna Mackenna 4860, Macul, Santiago, Chile;

${ }^{\mathrm{e}}$ University of Chicago, 640 S. Ellis Ave, Chicago, IL 60637;

${ }^{\mathrm{f}}$ Korea Astronomy and Space Science Institute (KASI) 776, Daedeokdae-ro, Yuseong-gu, Daejeon,

g Republic of Korea

Massachusetts Institute of Technology, Kavli Institute for Astrophysics and Space Research, 77 Massachusetts Ave., Cambridge, MA 02139;

${ }^{\mathrm{h}} \mathrm{UCO} /$ Lick Observatory, University of California, Santa Cruz, CA 95064

i Universidade de São Paolo, Rua do Matão 1226, 05508-900, São Paulo, Brazil

\begin{abstract}
The GMT-Consortium Large Earth Finder (G-CLEF) will be a cross-dispersed, optical band echelle spectrograph to be delivered as the first light scientific instrument for the Giant Magellan Telescope (GMT) in 2022. G-CLEF is vacuumenclosed and fiber-fed to enable precision radial velocity (PRV) measurements, especially for the detection and characterization of low-mass exoplanets orbiting solar-type stars. The passband of G-CLEF is broad, extending from $3500 \AA$ to $9500 \AA$. This passband provides good sensitivity at blue wavelengths for stellar abundance studies and deep red response for observations of high-redshift phenomena. The design of G-CLEF incorporates several novel technical innovations. We give an overview of the innovative features of the current design. G-CLEF will be the first PRV spectrograph to have a composite optical bench so as to exploit that material's extremely low coefficient of thermal expansion, high in-plane thermal conductivity and high stiffness-to-mass ratio. The spectrograph camera subsystem is divided into a red and a blue channel, split by a dichroic, so there are two independent refractive spectrograph cameras. The control system software is being developed in model-driven software context that has been adopted globally by the GMT. G-CLEF has been conceived and designed within a strict systems engineering framework. As a part of this process, we have developed a analytical toolset to assess the predicted performance of G-CLEF as it has evolved through design phases.
\end{abstract}

Keywords: Echelle spectrograph, precision radial velocity, G-CLEF, GMT, high dispersion spectroscopy, ELTs

\section{INTRODUCTION}

The design of The GMT-Consortium Large Earth Finder (G-CLEF) has been shaped by the requirement that the first-light instrument for the Giant Magellan Telescope (GMT) must enable a broad range of observational programs that can address

Ground-based and Airborne Instrumentation for Astronomy VI, edited by Christopher J. Evans, Luc Simard, Hideki Takami Proc. of SPIE Vol. 9908, 990822 - (c) 2016 SPIE · CCC code: 0277-786X/16/\$18 · doi: 10.1117/12.2233506 
the most urgent astrophysical problems. The scope of the G-CLEF science portfolio has been reviewed in previous publications ${ }^{1,2}$, including near-field cosmology, high- $Z$ cosmology and extragalactic astronomy. A particularly rapid shifting observational area is that of exoplanet science. To date, the catalogue of transiting exoplanet candidates has been dominated by the Kepler sample. This large catalogue generally consists of objects, which are faint and thus not amenable to studies of those exoplanet's atmospheres or precision radial velocity (PRV) mass determination of low-mass exoplanets, especially those that are Earth-twins. The Transiting Exoplanet Survey Satellite (TESS) ${ }^{3}$ and CHaracterising ExOPlanet Satellite (CHEOPS) ${ }^{4}$ will launch in the 2017-2018 time frame and provide a large sample of bright, low mass transiting exoplanets extending over the whole sky. While the current and coming generation of instruments on $3.5-10 \mathrm{~m}$ telescopes, especially ESPRESSO ${ }^{5}, \mathrm{CARMENES}^{6}, \mathrm{EPDS}^{7}, \mathrm{SHREK}^{8}, \mathrm{EXPRES}^{9}$ and Maroon$\mathrm{X}^{10}$, will open up new discovery space in the TESS and CHEOPS catalogues, many discoveries will only be possible with the $25.4 \mathrm{~m}$ aperture of the GMT, especially the search for the biomarker $\mathrm{O}_{2}$ in exoplanet atmospheres ${ }^{11,12}$.

A synoptic view of the G-CLEF subsystems is presented in Figure 1. G-CLEF is fiber-fed to isolate the spectrograph from gravitationally induced flexure and time-variable thermal gradients so as to enable PRV capability. G-CLEF is deployed on the azimuth platform of the GMT (see Figure 2 also). This makes it possible to connect the spectrograph to the telescope interface (the "front end") with extremely short, $17 \mathrm{~m}$ long optical fibers. This minimizes absorption of blue light and extends the blue end of the G-CLEF passband to very short wavelengths ( $3500 \AA)$ for stellar abundance studies, especially for the detection of iron complex feature just longward of $3500 \AA$. The passband of G-CLEF is specified to extend to $9000 \AA$ in the red, with a goal of $9500 \AA$. G-CLEF is instrumented with four different fiber feeds, which are enumerated in Table 1. The High-Throughput (HT) mode provides the lowest slit-loss with the largest diameter fiber, at the sacrifice of resolution. The Medium-Resolution (MR) mode is tuned to the requirements of stellar abundance studies at a resolution of 35,000. The Precision Radial Velocity (PRV) mode provides a resolution that resolves features of F, G and most K stars for the greatest possible RV precision. PRV mode has an integral mode scrambler, which reduces throughput by approximately $20 \%$ to reduce modal structure in the near and far field. Several high-Z cosmology programs also benefit from the high resolution of the PRV mode, but have no requirement for mode scrambling. To optimize G-CLEF for these programs we have an independent non-scrambled PRV (NS-PRV) mode which has higher throughput than the PRV mode. An interface to the multi-object spectroscopic feed $\left(\right.$ MANIFEST $^{13}$ ), to be provided by the Australian Astronomical Observatory, will provide multiplexed observational capability with G-CLEF at the same resolution of the MR mode.

Table 1: G-CLEF operating modes and corresponding resolutions are precision radial velocity (PRV), non-scrambled PRV (NS-PRV), medium resolution (MR), high throughput (HT) and multi-object spectroscopic (MOS) mode.

\begin{tabular}{|l|c|c|c|c|c|}
\hline Mode & PRV & NS-PRV & MR & HT & MOS \\
\hline Resolution & 108,000 & 110,000 & 35,000 & 19,000 & 35,000 \\
\hline
\end{tabular}

G-CLEF held a preliminary design review (PDR) in April 2015. The post-PDR optical design of G-CLEF is discussed elsewhere in these proceedings ${ }^{14}$. This may be compared with the pre-PDR design presented in an earlier publication ${ }^{15}$. The optomechanical design ${ }^{16}$ and software design ${ }^{17}$ are also presented in these proceedings. G-CLEF is being designed within a rigorous system-engineering framework that was presented in an earlier publication ${ }^{18}$. The larger context for the design of G-CLEF within the GMT instrumentation program is also presented elsewhere in these proceeedings ${ }^{19}$.

\section{SYSTEM DESIGN}

The system design shown in Figure 1 is broken down into:

1. The G-CLEF spectrograph (GCSPECT).

2. The G-CLEF Front End Assembly (GCFEA), which constitutes the telescope interface at the folded Gregorian focus of the GMT.

3. The G-CLEF Calibration Lamp System (GCCLS), which provides several different types of light sources for the calibrating G-CLEF.

4. The science optical fiber system (GCSFIB) that conveys light from the GCFEA to the GCSPECT.

5. The calibration light optical fiber system (GCCFIB), which delivers the calibration light to the input of the GCSFIB.

6. The interface to MANIFEST

Solid models of these subsystems appear in the two panels of Figure 2. 
The GCSPECT is an asymmetric white pupil design ${ }^{20}$ with two cameras dividing the spectrograph beam into a red and a blue channel at $5400 \AA$. The spectrograph is vacuum-enclosed to thermally isolated the spectrograph and stabilize the index of refraction of the immersing medium (see Figure 3). The vacuum chamber is thermally controlled with a thermometer/heater system integrated into thermal insulation panels. The core of this control system is the Isotech MicroK 125 temperature measurement system, which is capable of microKelvin accuracy. Since the spectrograph is deployed in the telescope dome, it is essential that it not produce thermal plumes which would compromise dome seeing. For this reason, the spectrograph is housed in a thermal enclosure that has a thermally stable interior $\left( \pm 0.5^{\circ} \mathrm{C}\right)$, yet does not dissipate more that $100 \mathrm{~W}$ into the telescope chamber. This is accomplished with a thermal scavenge plenum that convects heat in the outer layer of the thermal enclosure away with the telescope heat exhaust system.

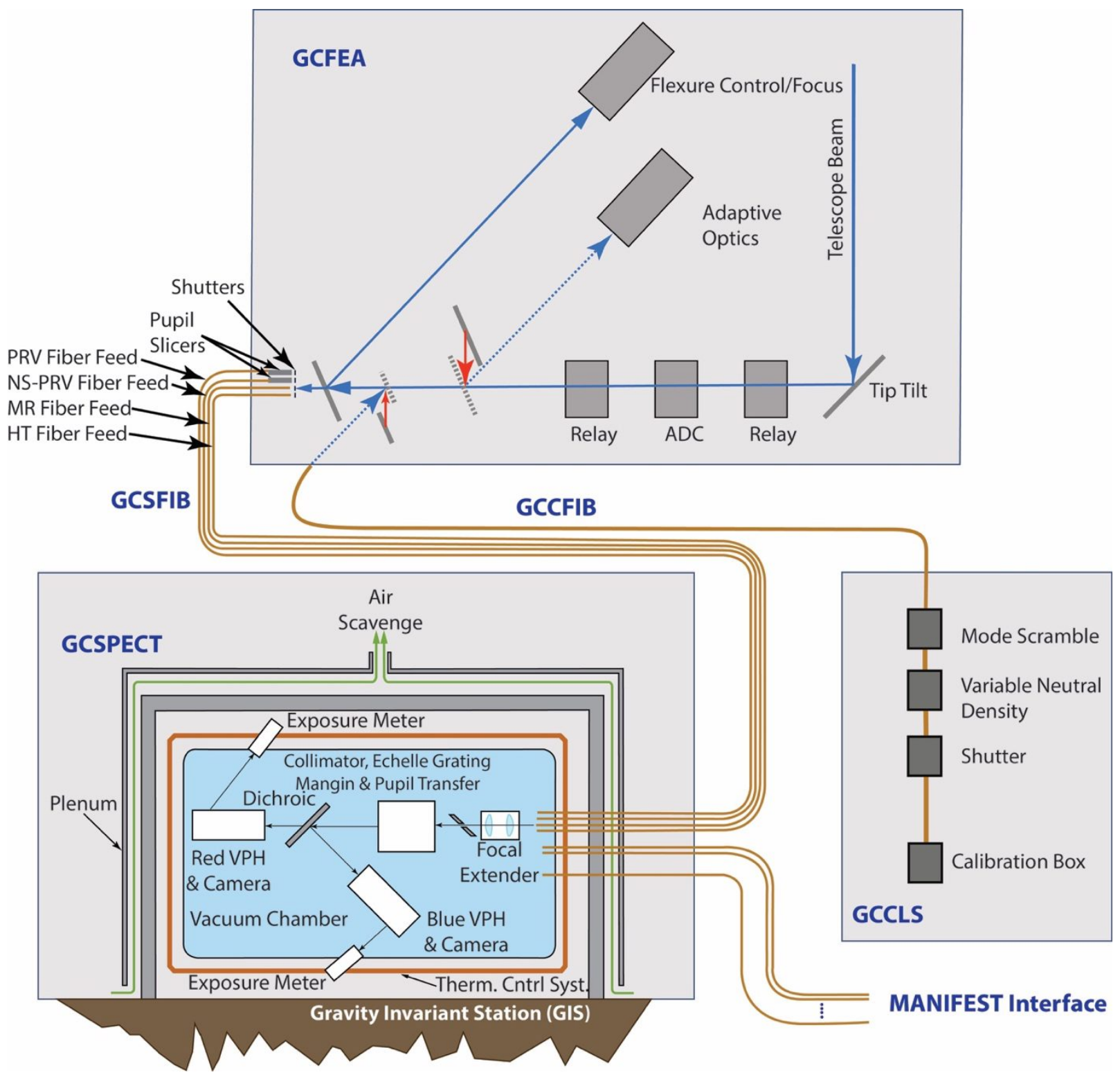

Figure 1:G-CLEF system diagram showing the major subsystems. 
The telescope interface - the GCFEA - performs several functions. All natural seeing instruments on the GMT are required to both guide and focus themselves. The GMT will position and guide itself with 0.2 arcsecond accuracy over the observable sky with the Acquisition Guide Wavefront Sensing Subsystem (AGWS $)^{21}$. However, flexures and deformations that are not common mode between the optical path of the AGWS and G-CLEF require compensation. This compensation is accomplished with the G-CLEF Flexure Control Camera (FCC), which is the subject of a separate contribution to these proceedings $^{22}$, and is discussed briefly in Section 3.4 of this paper. The FCC also maintains focus at the G-CLEF telescope focal plane. The error signal that FCC generates is used to servo the position of a tip-tilt mirror that serves as the G-CLEF fold/tertiary. Flexure, elevation dependent deformation and thermal expansion have large time-constants, so the sampling time of the FCC control loop is extremely long. Since it is very important to minimize the length of the science optical fibers, the telescope focus is relayed to the edge of the telescope instrument platform by a pair of deployable triplet lenses. Atmospheric dispersion compensation (ADC) is integral to this relay system. These optics have been described in an earlier publication ${ }^{1}$.

Fiber selection is another function that is performed by the GCFEA. The fiber selector also can inject various calibration light sources into each of the science fibers. An additional position on the fiber selector accommodates the GCFEA alignment optics.

Modeling has shown that the GMT adaptive optics system can improve both the near-field stability within the spectrograph and throughput, in some cases by a factor of $2^{1}$. A provision exists to upgrade the GCFEA to accommodate a wavefront sensor when adaptive optics are implemented on the GMT some time after first light ${ }^{23}$.
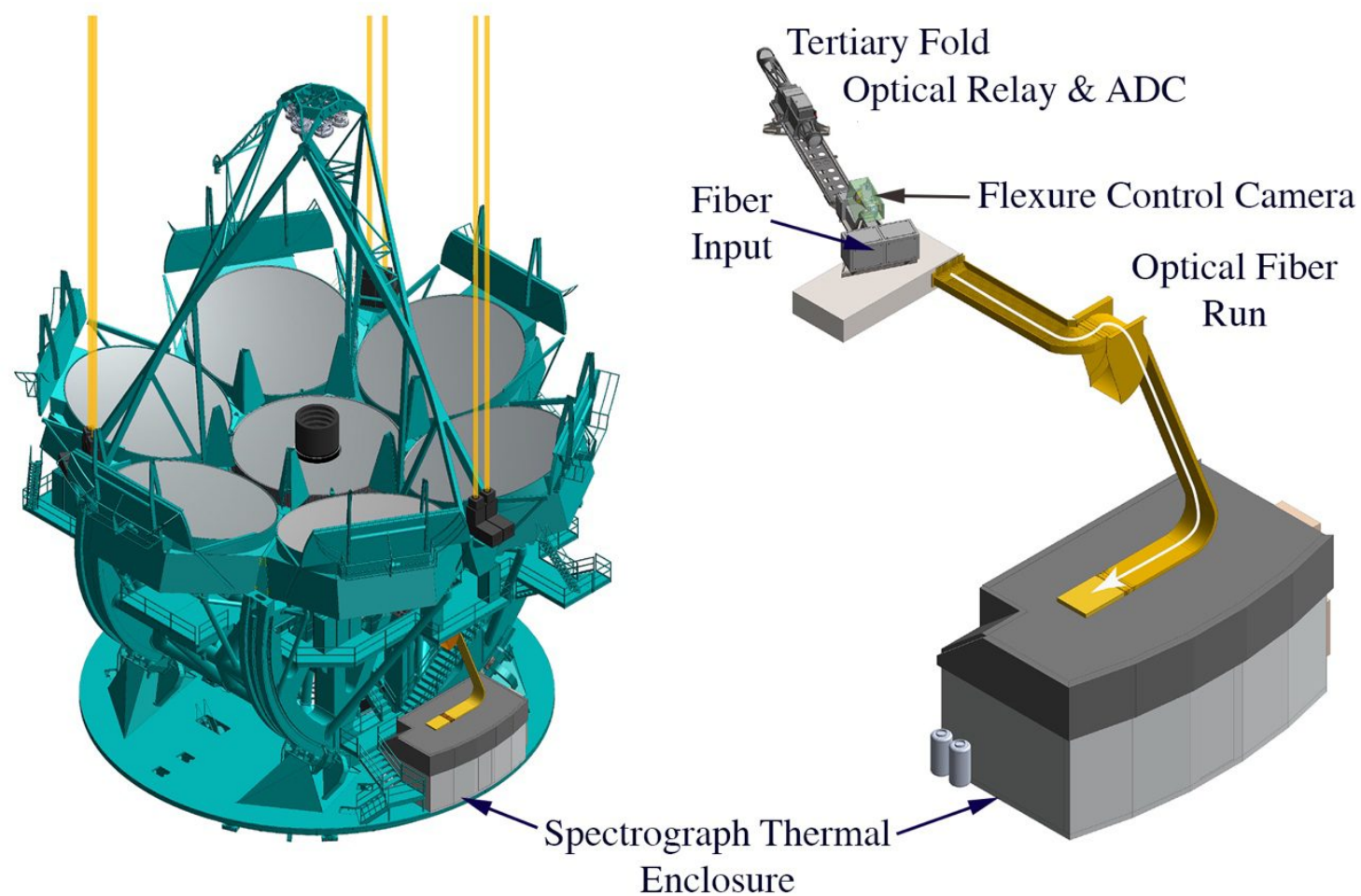

Figure 2: Left Panel - G-CLEF in mounted on the gravity-invariant azimuth platform of the GMT. Laser guide star beams for adaptive optic correction are shown. Right Panel - The telescope interface to the G-CLEF which consists of the tertiary fold, the relay optics and science optical fiber run. A flexure control system in implemented at the start of the optical fiber run.

The GCSFIB connects the GCFEA to the spectrograph and the GCCFIB connects the calibration system - the GCCLS to the GCSFIB input. The GCCLS contains several internal calibration lamps, and will be fed by several external light sources. The GCCLS also contain the calibration system shutter and a continuously adjustable neutral density filter to 
control the intensity of light delivered to the input of the science fibers. Since variable modal structure degrades RV precision, a mode scrambler is included in the GCCLS.

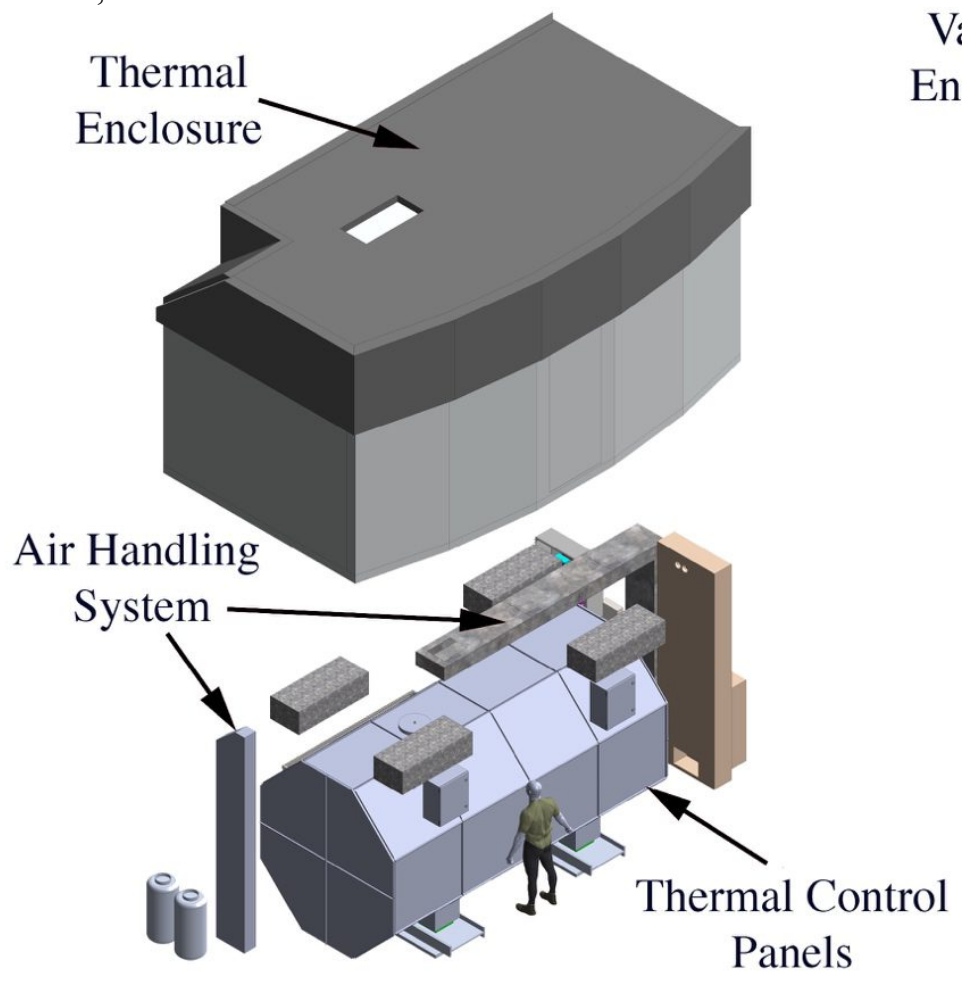

Vacuum

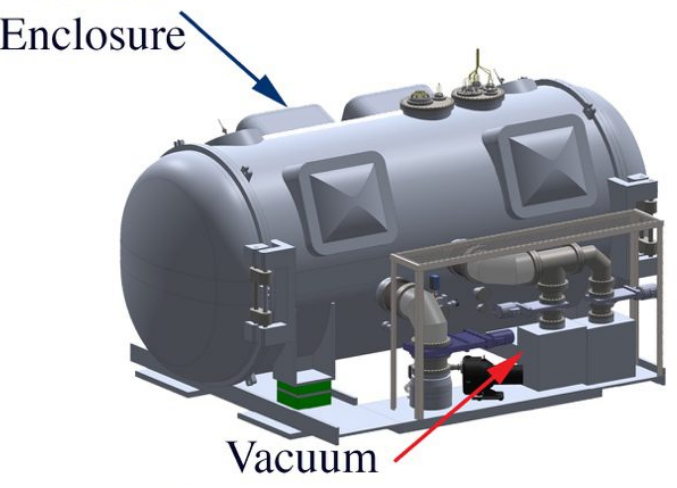

Pump System

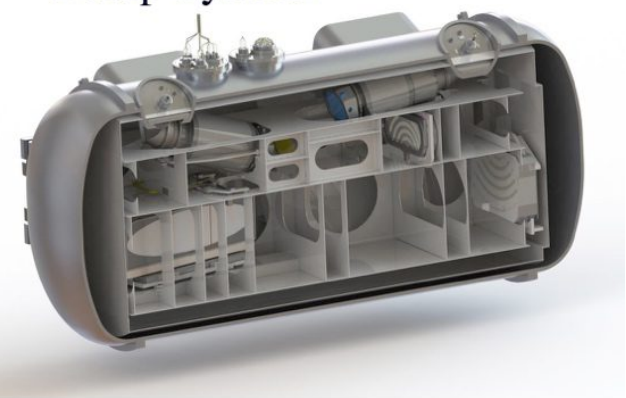

Figure 3:Left Panel: Spectrograph thermal control and air circulation system. Right panel: Spectrograph vacuum enclosure.

\section{OPTICAL DESIGN}

\subsection{Spectrograph Optical Design}

The post-PDR spectrograph optical layout appears in Figure 4 and the optical properties of the spectrograph are presented in. The optical design was reviewed at the PDR and several recommendations were made to optimize and improve the PDR optical design. In response to these recommendations, we made the following modifications to the optical design:

- We have reduced the camera lens count by allowing one element to be aspheric in each camera. We had previously tried to mitigate lateral color in the camera optical design to make it possible to test the cameras in white light. For the post-PDR design we relaxed this requirement to help reduce the lens count.

- We eliminated a pernicious first-order ghost produced by the Mangin mirror that was identified at PDR.

- We have reduced the focal length of the camera optics from $500 \mathrm{~mm}$ to $450 \mathrm{~mm}$ to reduce the plate scale and permit an increase in the inter-order spacing in the echellogram

- We have redesigned the input focal ratio adjustment optics to produce a real, rather than virtual, slit image which permits scattered light control.

We also did an in-depth ghost analysis, focusing in particular on "Littrow ghosts" produced by the volume phase holographic (VPH) grating ${ }^{25}$. These investigations prompted us to change the blue camera VPH from a pure VPH grating to a VPH grism. As a part of this redesign, we identified the VPH zero order beam as an opportunity to implement the exposure meter. The exposure meter is used to determine the photocenter of an integration, so as to improve the accuracy of the barycentric correction. At the recommendation of the PDR committee, we have added mild dispersion to both exposure meters to measure the color dependence of the photocenter arising from imperfect atmospheric dispersion compensation. 
A more detailed discussion of the spectrograph optical design is presented elsewhere in these proceedings ${ }^{14}$.

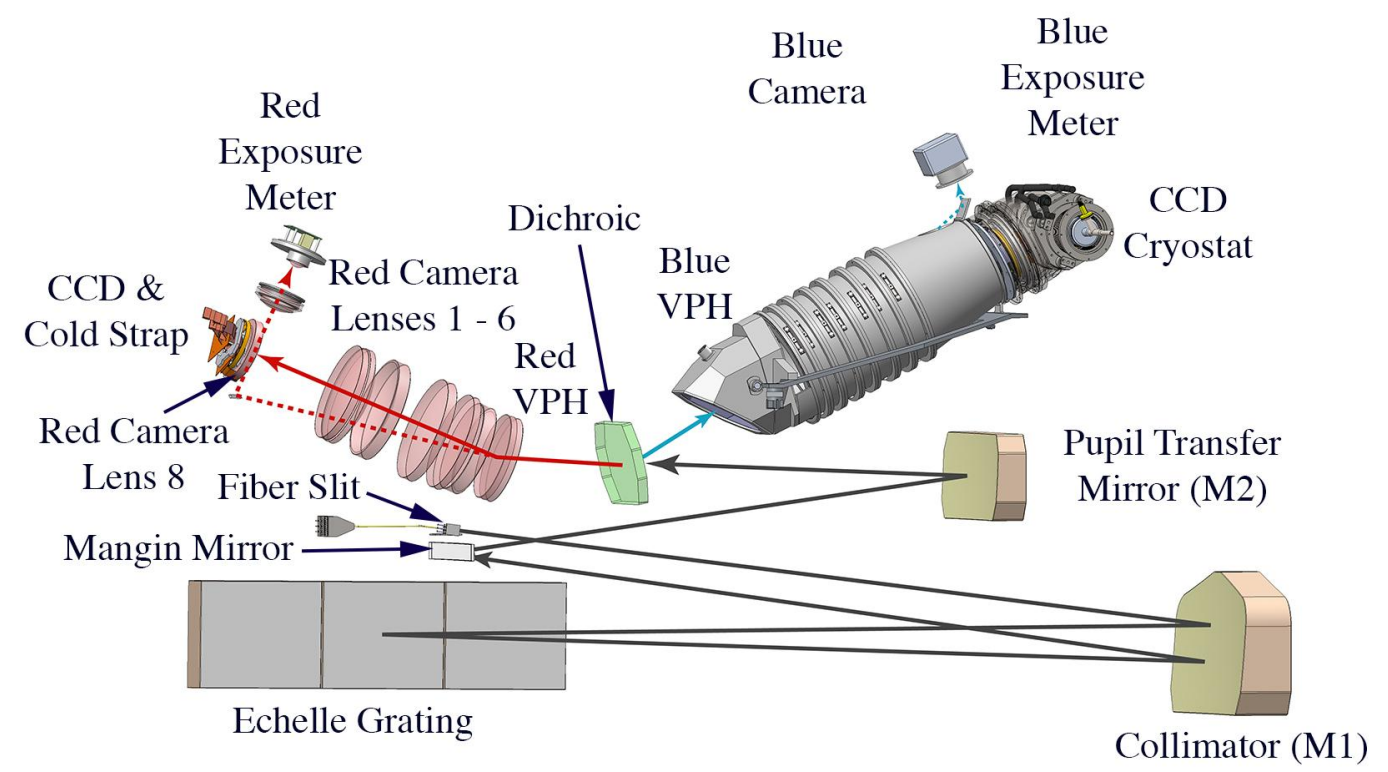

Figure 4: The spectrograph optical design. The spectroscopic light path is shown in a solid line. The zero order path, i.e., light undeviated by the VPH, is shown with a dotted line. The red camera optical elements are shown, while the blue camera optic mount is shown.

Table 2: Summary of G-CLEF spectrograph parameters. We have adopted a "baseline" pixel size, which will ultimately depend on which vendor is chosen to supply the spectrograph focal plane.

\begin{tabular}{|l|c||c|c|}
\hline G-CLEF Property & Value & G-CLEF Property & Value \\
\hline Spectrograph Beam Diameter & $300 \mathrm{~mm}$ & Spectrograph Focal Ratio & $\mathrm{F} / 8$ \\
\hline Echelle Grating Facet Count & 3 & Collimator focal length & $2400 \mathrm{~mm}$ \\
\hline Fiber Output Focal Ratio & $\mathrm{F} / 3$ & Pupil Xfer focal length & $1600 \mathrm{~mm}$ \\
\hline Camera Beam Diameter & $200 \mathrm{~mm}$ & $\begin{array}{c}\text { Pup.Xfer Mirr./Cam. } \\
\text { Reduction }\end{array}$ & $\begin{array}{c}450 / 1600= \\
0.2813\end{array}$ \\
\hline Camera Focal Ratio & $\mathrm{F} / 2.25$ & PRV Slit Image Size & $0.075 \mathrm{~mm}$ \\
\hline Camera Focal Length & $450 \mathrm{~mm}$ & Baseline Pixel Size & $0.010 \mathrm{~mm}$ \\
\hline Passband & $\begin{array}{c}3500 \AA-5400 \AA \& \\
5400 \AA-9500 \AA\end{array}$ & Camera lens count (blue/red) & $8 / 7$ \\
\hline
\end{tabular}

\subsection{Science and Calibration Fiber System (GCSFIB \& GCCFIB) Optical Design}

The system design of the GCSFIB and GCCFIB are shown in Figure 5. The physical implementation of the input end is shown in Figure 6.

The GCSFIB consists of four set of fibers for the four different observing modes - HT, MR, PRV and NS-PRV. Each mode has two sky fibers, only one of which is used for any given observation. The parameters defining these modes are given in Table 3. All multimode optical fiber used in G-CLEF will be Polymicro FBP, chosen for its superb broad-band transmissivity. The fiber mode is selected by linear translation of the fiber slits. The $\mathrm{f} / 8$ telescope beam passed through small holes in the slit mirrors. In the case of the HT and MR mode, the focal ratio is adjusted to $\mathrm{f} / 3$ by a doublet lens cemented to the fiber end. In the case of the PRV and NS-PRV modes, it is necessary to slice the telescope pupil achieve the resolution required (specification $\mathrm{R} \geq 100,000$ ) for these modes. Immediately after the slit mirror, the telescope beam is 
collimated to form a pupil image which is then sliced into seven subapertures that map onto the seven individual telescope primary apertures. Each fiber slit (sky or science) has an independently operable shutter.

Table 3: G-CLEF Science fiber parameters.

\begin{tabular}{|l|c|c|c|c|c|}
\hline Mode & Resolution & Fiber & \# Science Fibers & $\begin{array}{c}\text { \#Cal/Sky } \\
\text { Fibers }\end{array}$ & $\begin{array}{c}\text { Resolution } \\
\text { element (pixels) }\end{array}$ \\
\hline PRV & 108,000 & Hexagonal $100 \mu$ Core & 7 & 2 & 7.5 \\
\hline NS-PRV & 108,000 & Circular $100 \mu$ Core & 7 & 2 & 7.5 \\
\hline MR & 35,000 & Circular $300 \mu$ Core & 1 & 2 & 20 \\
\hline HT & 19,000 & Circular $450 \mu$ Core & 1 & 2 & 30 \\
\hline MOS & 35,000 & Circular $300 \mu$ Core & 40 & $\mathrm{n} / \mathrm{a}$ & 20 \\
\hline
\end{tabular}

Calibration light is produced in the remote GCCLS and conveyed to the GCFEA by the GCCFIB. The light is delivered to the science fibers by one of two calibration probes that can be positioned independently in front of arbitrary science fibers or fiber bundles (i.e. the PRV and NS-PRV modes). The optical design of these probes is shown in the left panel of Figure 6. Any pair of calibration light sources can be delivered to any fiber slits, including fiber slits that are not being used for observation at that time.

\section{Fiber Subsystem Schematic}

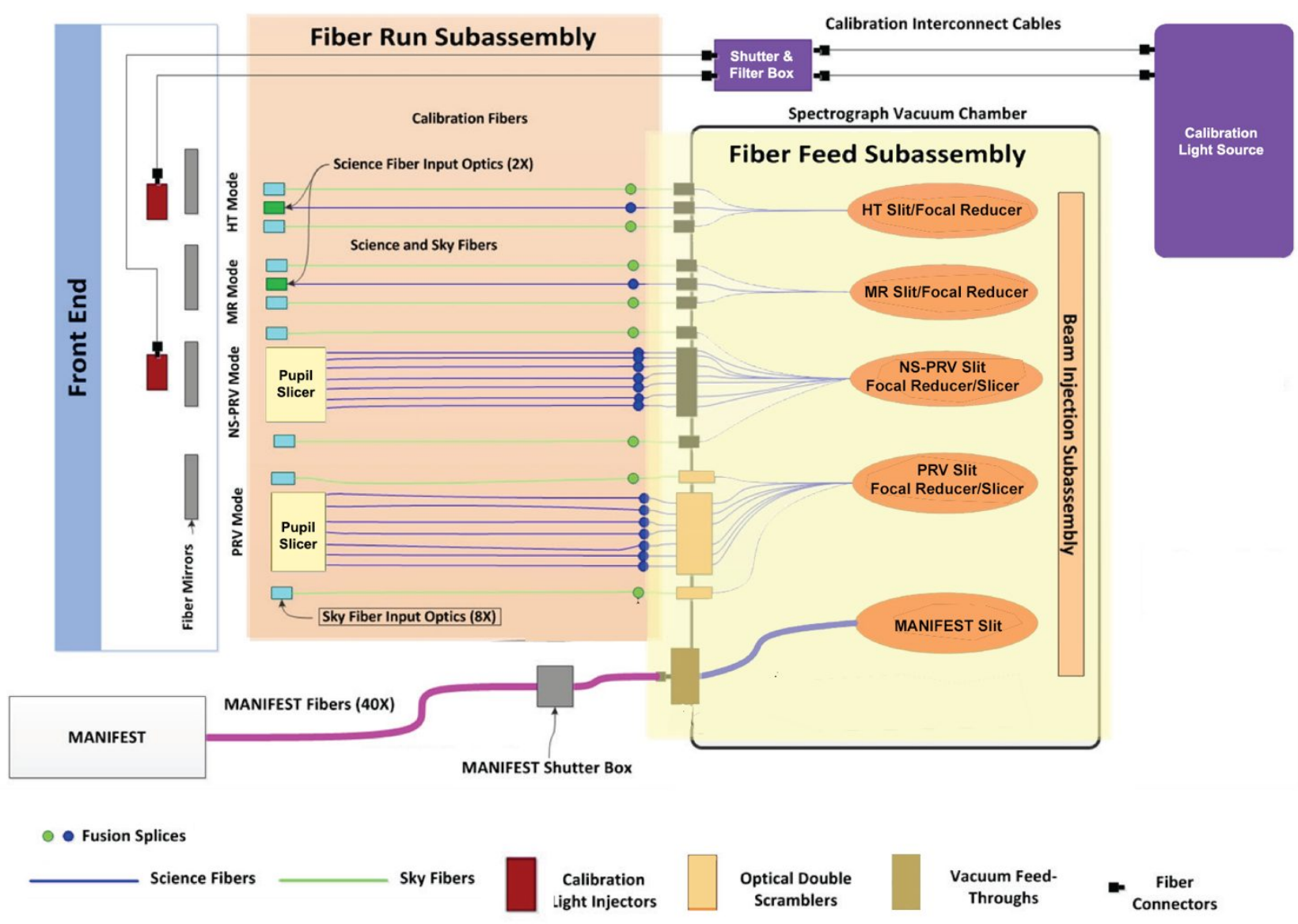

Figure 5: System design of the GCSFIB and the GCCFIB. 
Wherever possible, fiber interfaces have been connectorized using standard fiber connectors used in telecommunication. However, for the fibers in the GCSFIB, connectors tend to reduce throughput and introduce modal structure that is unacceptable. The G-CLEF team will fuse the fiber runs to flying leads of the fibers that pass through the spectrograph vacuum vessel fiber feed-throughs with a 3SAE S183PMII-V2 Advanced Fusion Splicer optical fiber splicer. This is a risk mitigation against fiber breakage or damage, vastly simplifies deployment of the GCSFIB and offer the potential to replace the GCSFIB fibers commercially available at first light with higher performance optical fiber that may become available in the future. Extensive laboratory testing and prototyping has demonstrated that the throughput and focal ratio degradation in a spliced fiber in indistinguishable from fiber that has not been spliced.

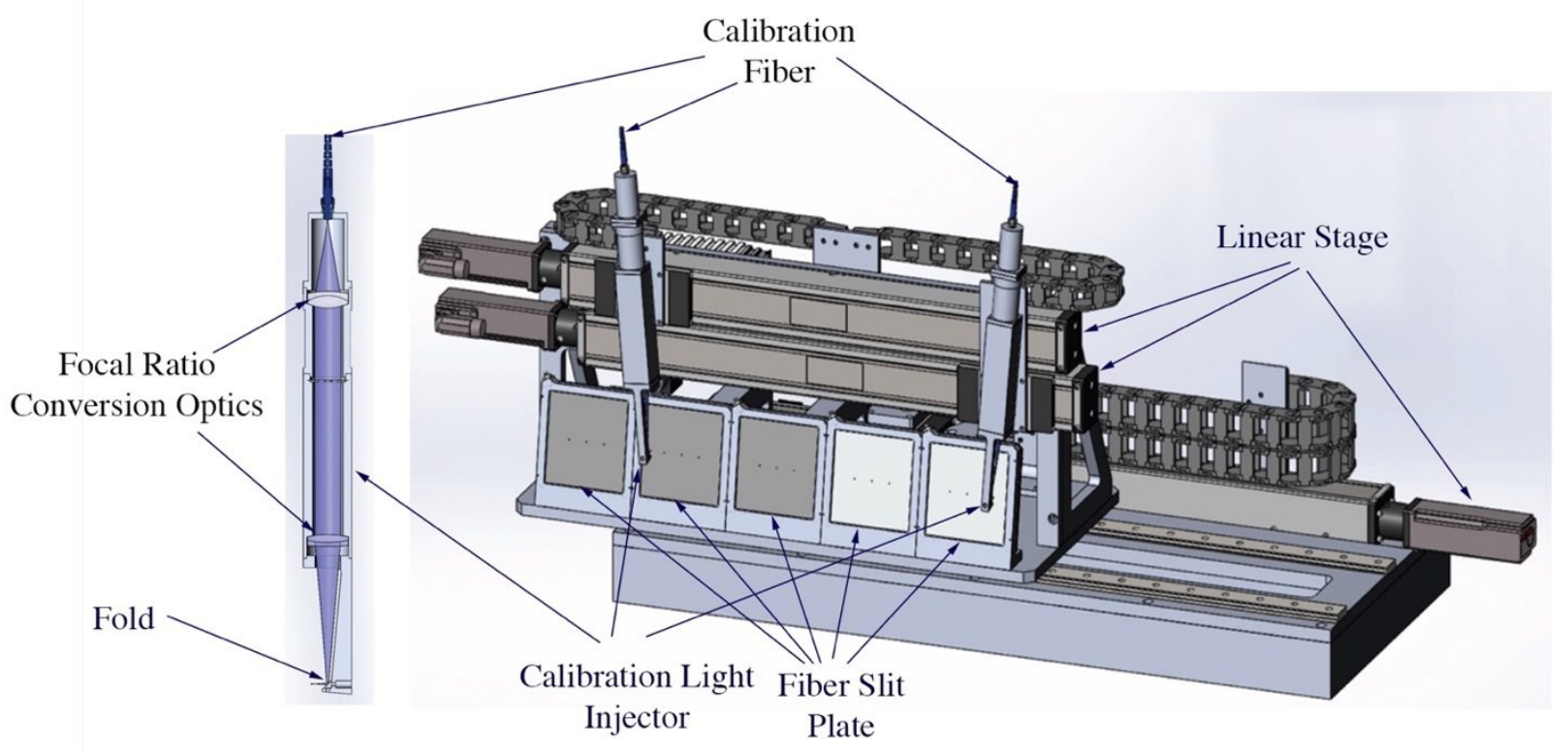

Figure 6: Physical implementation of the fiber feed/selector. A section view of a calibration light injector is shown on the left panel.

The vacuum feed through for the NS-PRV, MR and HT vacuum feedthroughs will be implemented by potting the fiber in epoxy wells machined into vacuum flanges. The PRV vacuum feedthrough will be integrated with the mode scrambler so the mode scrambler will also function as the vacuum feedthrough.

\subsection{Calibration Lamp System (GCCLS) Optical Design}

The calibration system for G-CLEF is based on the design of the HARPS and HARPS-N. However, the primary wavelength scale calibration for the HARPS instruments was done with metallic cathode thorium argon hollow cathode lamps. At present, no manufacturer produces these lamps and the global supply is exhausted. The thorium oxide lamps that are available have impurities that severely compromise their utility for PRV calibration. The PRV community is currently experimenting with several alternative calibrators, especially laser frequency combs (LFCs) ${ }^{25,26}$, passively stabilized etalons ${ }^{27}$ and actively stabilized etalons ${ }^{28}$. The design of the calibration system was based on the assumption that the optical system would inject an image of a hollow cathode lamp or diffused incandescent bulb into the input fibers of the GCCFIB.

Energetiq $^{\mathrm{TM}}$ laser-driven light sources deliver bright, uniform continuum light across the entire G-CLEF passband. These light sources vastly outperform incandescent lamps as continuum calibrators. Incandescents have very weak blue output. Deuterium lamps which have boosted blue output, also have highly structured spectra. Hollow cathode lamps and incandescent bulbs are compact, thus can be housed within the GCCLS. However, all the new calibration light sources are large and will need to be mounted independently. It is likely that most of the light sources in the GCCLS will be integrating spheres fiber fed by external unit. 
The optical layout of the of the GCCLS injection system is shown in Figure 7. The 3mm diameter light source is collimated by an achromatic doublet. The parallel beam is imaged onto each of two calibration fiber inputs. The beam can feed either of two calibration fiber inputs, or both calibration inputs at the same time. Light sources are selected by positioning a calibration fiber in front of a particular calibration light source. A dark position provides a shutter for the calibration fibers.

One observing mode is to simultaneously feed a sky fiber with a wavelength calibration during a science integration in the science fiber (the simultaneous calibration technique) ${ }^{29}$. For this reason, it is essential that the light level be adjusted for different integration times. This is accomplished with an in-line, continuously variable circular neutral density filter that can be rotated to select an illumination level to provide adequate calibration light signal to noise driving the detector into saturation.

Calibration box Unit: mm
Lens 2

Thorlab AC080-020-A

$\mathrm{f}=19.96 \mathrm{~mm}, \mathrm{D}=8 \mathrm{~mm}$

Lens 1

Befort Wetzlar 925-1250

$\mathrm{f}=125 \mathrm{~mm}, \mathrm{D}=25 \mathrm{~mm}$

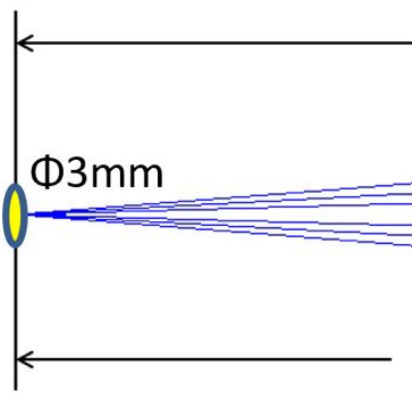

205.136

Figure 7: The optical layout of the GCCLS injection system.

\subsection{The flexure control camera (FCC)}

The optical layout of the FCC is shown in Figure 8. In addition to flexure control, the FCC is used to control focus at the slit mirror. Focus is determined with a tent prism that divides a pupil internal to the FCC. Focus error signals are sent to the telescope control system, while flexure corrections are used to servo the G-CLEF tip-tilt tertiary.

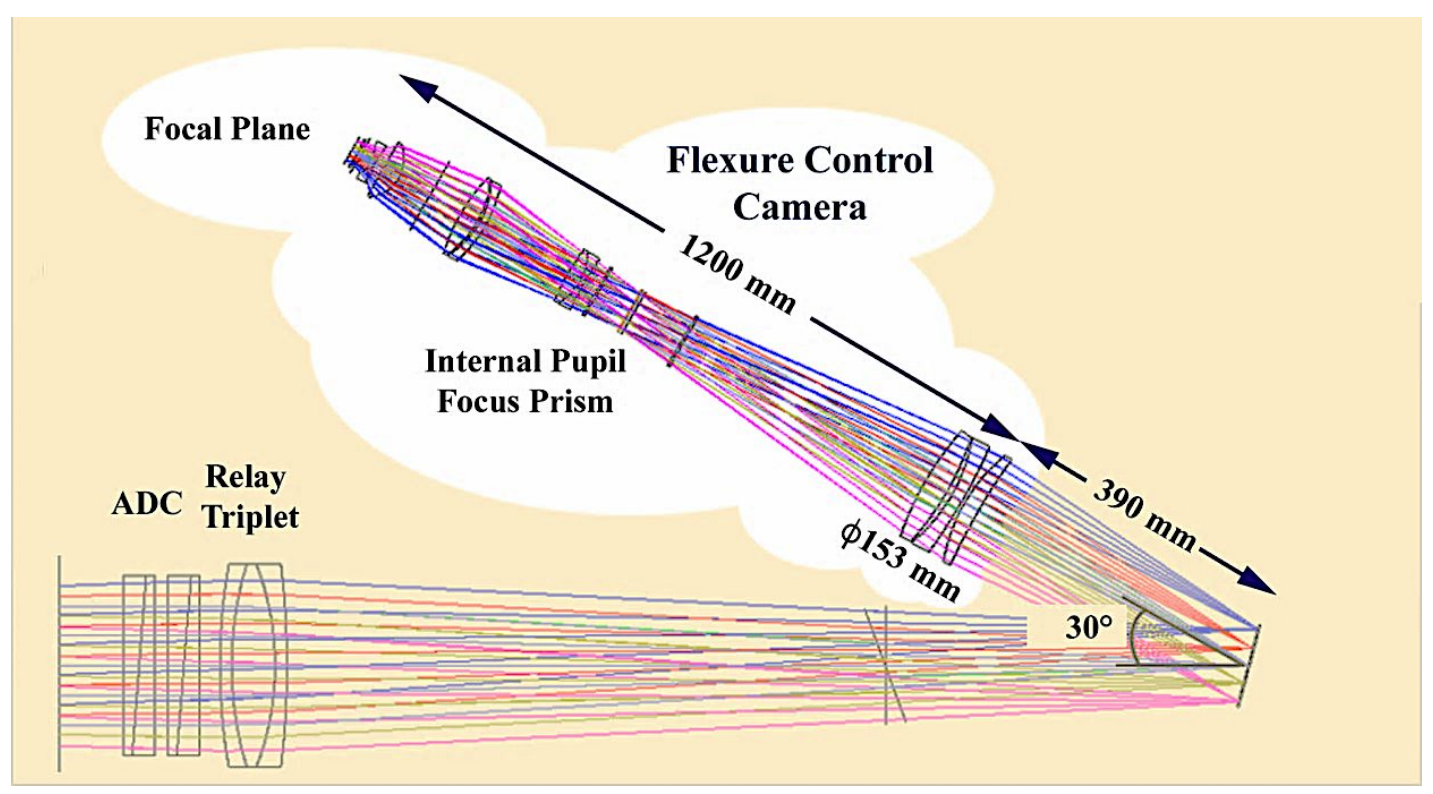

Figure 8: The G-CLEF FCC optical layout. 
The FCC optical design consists of a triplet, which forms the intermediate focus. Slit image is then produced at the focal plane of the FCC with three pairs of lenses. The diagonal field of view on the CCD is 60 arcminutes. Field rotation of the telescope is removed by rotating the entire CCD camera. A considerably more detailed description of the FCC can be found elsewhere in these proceedings ${ }^{22}$.

\section{MECHANICAL DESIGN}

\subsection{Spectrograph Mechanical Design}

The G-CLEF optical bench mechanical design is shown in Figure 9. A section view of the G-CLEF spectrograph mounted in the vacuum enclosure appears in Figure 10. Further detail concerning the optomechanical design of the spectrograph can be found in another submission to these proceedings ${ }^{16}$. The vacuum chamber is fabricated of aluminum and considerable effort has gone into designing access ports to allow assembly, alignment and service. The vacuum is established with a deployable, high capacity pump station and maintained with ion pumps, which run continuously.

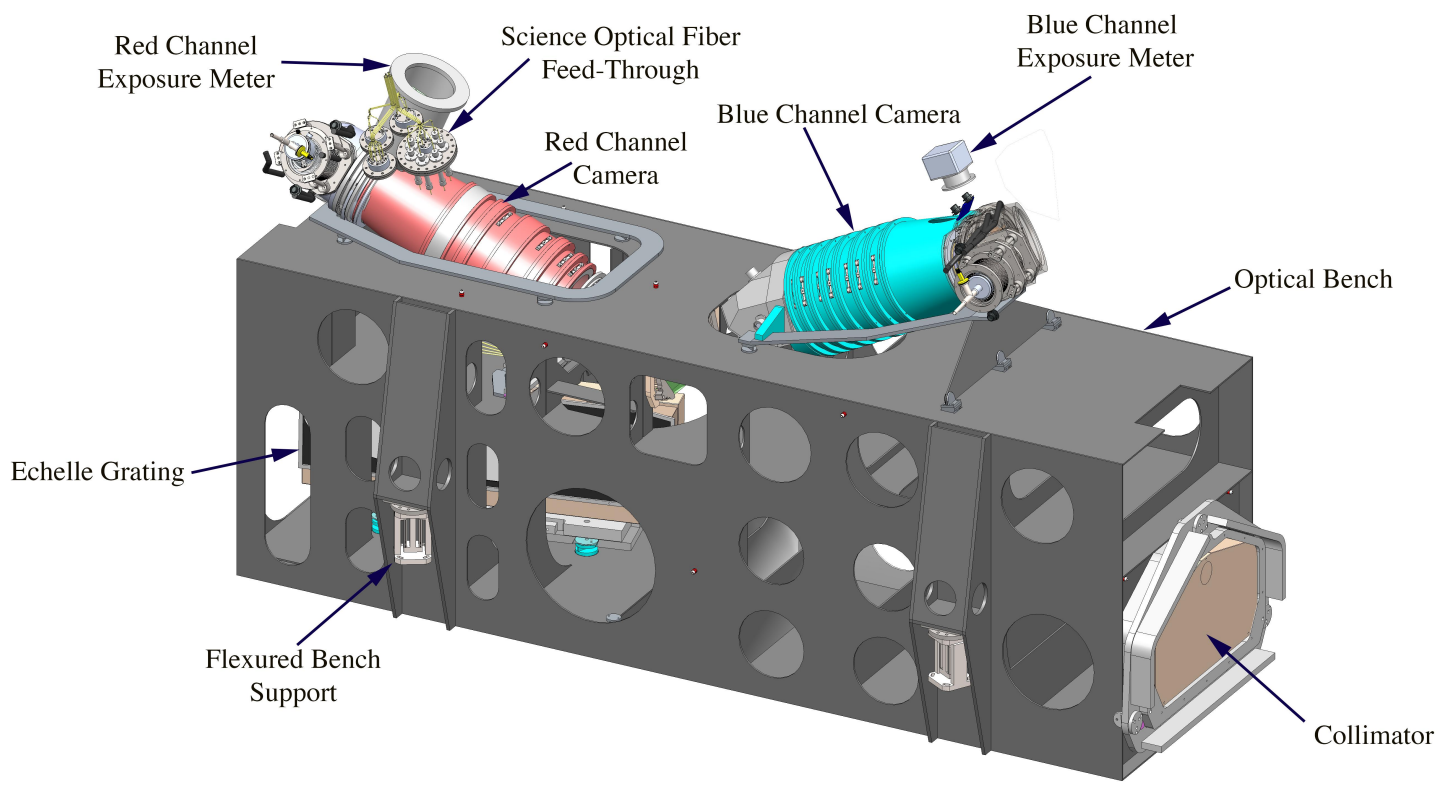

Figure 9. Major elements of the spectrograph mechanical design.

The optical bench is made of carbon fiber epoxy composite with Invar interfaces to the optic mounts. The optical bench is supported semi-kinematically on three titanium blade flexures. An extensive trade study was made comparing various possible materials for the optical bench, i.e. composite, Invar and mild steel. Factors in this study were weight, cost, and requirements on the thermal control system to achieve the specification RV precision. The fact that G-CLEF is mounted on the moving mass of the telescope, i.e., the azimuth platform, and is quite large, hence quite massive, made weight a significant trade factor. Further detail on the optical bench design, trade study and analyses supporting that study can be found in Mueller ${ }^{16}$ and Baldwin ${ }^{30}$ in these proceedings.

The specification performance requirements for G-CLEF exceeded the current state-of-the-art, especially in the area of RV precision, so it was necessary to prototype several subsystems to verify that subsystem requirements could be met and validate various performance budgets. As Mueller ${ }^{16}$ discusses, two critical areas were the thermal control of the spectrograph itself and the cold head which maintains the science CCDs at cryogenic operating temperature. Another critical area was that of co-alignment of the echelle grating facets. The largest surface relief diffraction grating available is a $300 \mathrm{~mm} \times 400 \mathrm{~mm}$ facet produced by Richardson Gratings, the only manufacturer of replicated, large format surface relief diffraction gratings. The G-CLEF beam size and required resolution drove us to an echelle design with a triple mosaic of these gratings to form a $300 \mathrm{~mm} \times 1200 \mathrm{~mm}$ grating at a Littrow blaze angle of $76^{\circ}$ (i.e., R4). Newport has already developed a process to mosaic two large format grating onto a monolithic Zerodur substrate. However, after an extensive dialogue with the Richardson management and technical staff, it was determined that the necessary risk reduction 
exercises that would be required for Richardson to deliver a triple-monolith grating would exceed the cost envelope of the G-CLEF program. For that reason, the G-CLEF team adopted a design that mechanically metered the three echelle facets to form a triple mosaic. This had been the approach taken by the HIRES ${ }^{31}$ team, however HIRES was not designed as a PRV instrument, so the alignment accuracy and stability requirements were considerably relaxed compared with G-CLEF. The ESPRESSO ${ }^{32}$ instrument for the VLT has taken a similar approach, however the ESPRESSO instrument has not yet had first light, so performance data for that design is not yet available.

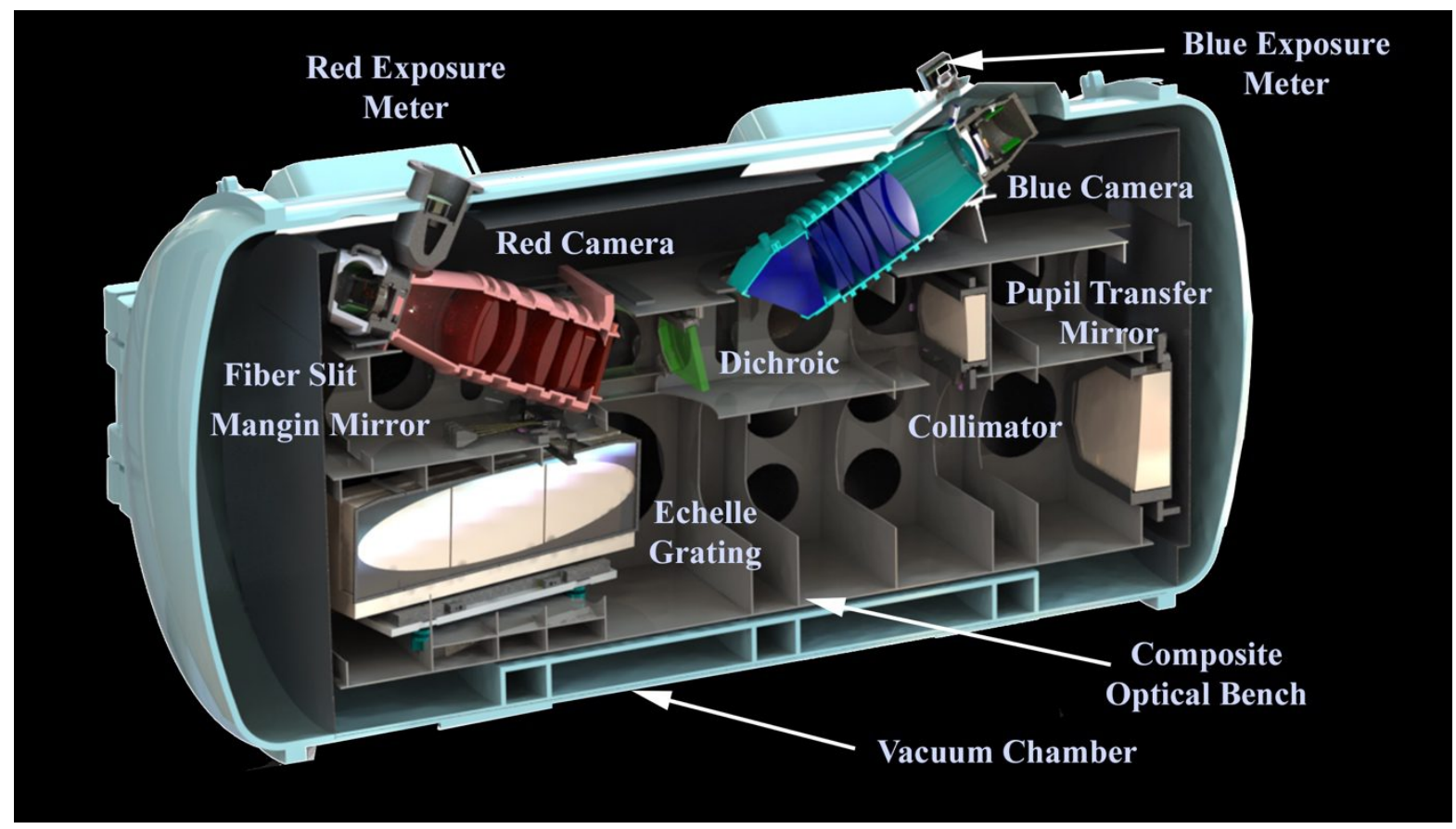

Figure 10: Section view of the G-CLEF spectrograph mounted in vacuum chamber.

The design of the echelle mosaic is shown in the left panel of Figure 11.The grating facets themselves have details machined into the Zerodur substrate that allow preloads to pull each facet into alignment on adjustable shim. The overall metering structure is a machined Zerodur monolith which mounts on the composite optical bench with Invar interfaces.

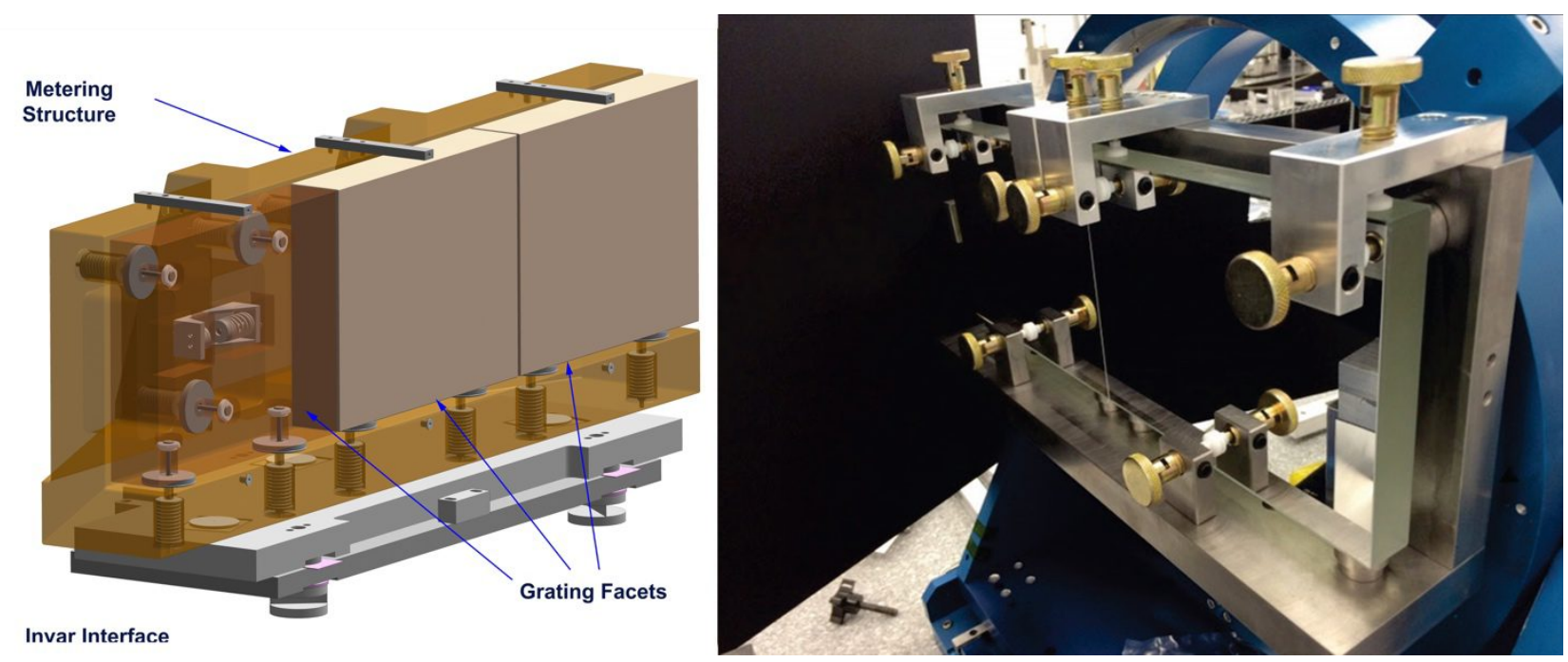

Figure 11: Left panel: Mechanical mounting and metering structure of the three echelle grating facets. The rightmost fact is shown as transparent to expose the facet mounting scheme. Right panel: Prototype of the echelle grating mount and metering structure. 
The requirement for the angular alignment of the facets is quite demanding ( $\pm 1 \operatorname{arcsec})$, so the G-CLEF team decided a prototype was necessary for risk reduction of this subsystem ${ }^{16}$. The prototype is shown in the right panel of Figure 11 . The facet surrogates are fabricated out of fused silica and the metering structure is mild steel. The alignment of the facet surrogates has been measured interferometrically. At present the alignment accuracy is somewhat above the requirement, but when proper account is taken for the relatively high coefficient of thermal expansion of the facet surrogates and metering structure, as well as the geometric scale differences between the prototype and the actual mosaic, the alignment methodology is sufficient to support the overall G-CLEF performance requirements. The prototype continues to be valuable as a testbed for optimization of shim design.

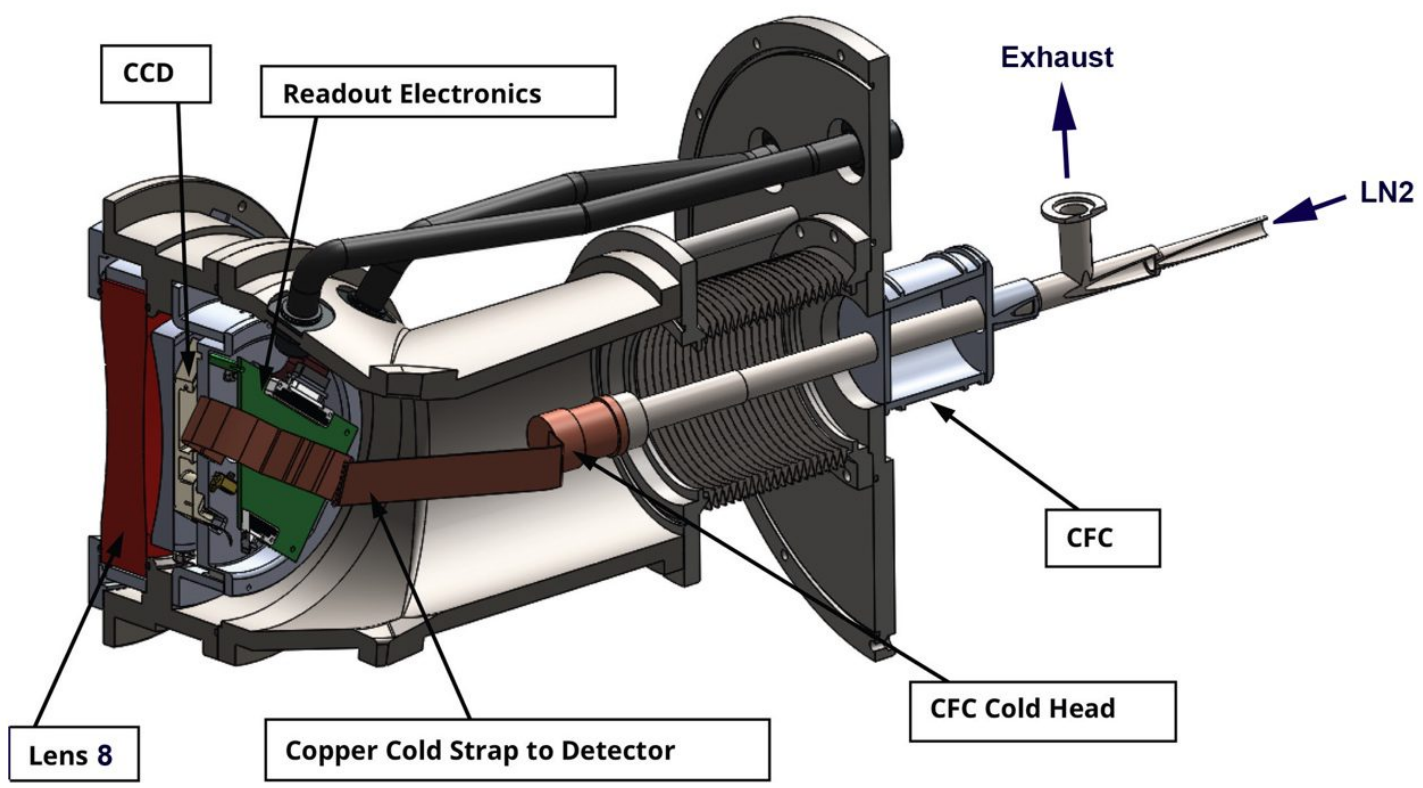

Figure 12: Red camera CCD cryostat in section.

Thermal stability of the CCD focal plane is another area of focus in the design of PRV-capable spectrographs. The technical issues attending focal plane thermal stability are two-fold. The cold head that refrigerates the focal plane must be well temperature-stabilized if the focal plane itself is to be thermally stable. The other issue is that of changing heat dissipation distribution within the CCD itself, which depends on whether the CCD is integrating or reading out. While the dissipation can be equilibrated somewhat by running the serial registers at all times, the parallel registers are only exercised during read out, increasing overall heat dissipation during read out and changing the spatial distribution of the internal CCD heat dissipation.

Considerable progress has been made on the design of extremely stable cold heads by other instrumentation groups ${ }^{31}$. However, our team found it expeditious to collaborate with Janis Research to develop an ultrastable cold head. Janis indicated they could deliver commercial, off-the-shelf continuous $\mathrm{LN}_{2}$ flow cold heads with remarkable thermal stability. Preliminary testing suggests long term stability at the $4 \times 10^{-3} \mathrm{~K}$ level is easily achieved.

The problem of varying thermal dissipation at the CCD is more complicated and analysis continues to optimize the focal plane heater system geometry to best mitigate this effect. An alternative approach to this problem is to exploit the oversampling of even the smallest resolution element (PRV mode - 7.5 pixels) and simply run the parallel register continuously, shifting the charge back and forth between pairs of pixels. Preliminary optical modeling indicates that the degradation in the PSF is undetectable. We expect to test this mode operationally late in the current Phase C of the GCLEF project.

A sectioned view of the red camera is shown in Figure 12. The blue camera cryostat is essentially identical. The cold head is coupled to the science focal plane by a copper strap. The last element of both cameras is mounted on the cryostat and 
serves as a vacuum window when the cryostat is operated in air for testing and integration. In operational mode, the cryostat is hard-mounted to the camera lens barrel. It must be mechanically decoupled from the spectrograph vacuum vessel to prevent coupling vibration or deformation of the vacuum vessel due to changing pressure differentials when the vessel is pumped out or as ambient atmospheric pressure changes.

While the spectrograph is maintained at vacuum, it is necessary to isolate the evacuated volumes of both CCD cryostats, so each cryostat has an independent vacuum system. This puts special constraints on the mounting of the last camera lens. Several configurations are possible for the cryostat. During testing, the exterior of the cryostat will be at atmospheric pressure (i.e., STP), and the interior will be at vacuum. In operational mode, both exterior and interior will be at vacuum. Finally, the failure mode where the cryostat vacuum is lost, but the spectrograph is still vacuum enclosed must be accommodated. The lens/vacuum window must be at its prescribed axial position to perform optically in the first two of these configurations. We have adopted a solution that employs an opposed, double O-ring mount, where the lens is continuously registered axially in a vacuum/vacuum or STP/vacuum configuration, but still forms a vacuum seal in a vacuum/STP configuration, the lattermost configuration resulting from a cryostat vacuum failure.

\subsection{Optical Fiber Mechanical Design}

At the interface to the GCFEA optical relay there are two types of science fiber interface - the MR/HT fiber feed, which consists of three identical fibers, and that of the PRV/NS-PRV fiber feed with a pair single sky fibers and seven pupilsliced science fibers. The two calibration fibers must also be accommodated. The design of these interfaces is illustrated in Figure 13.

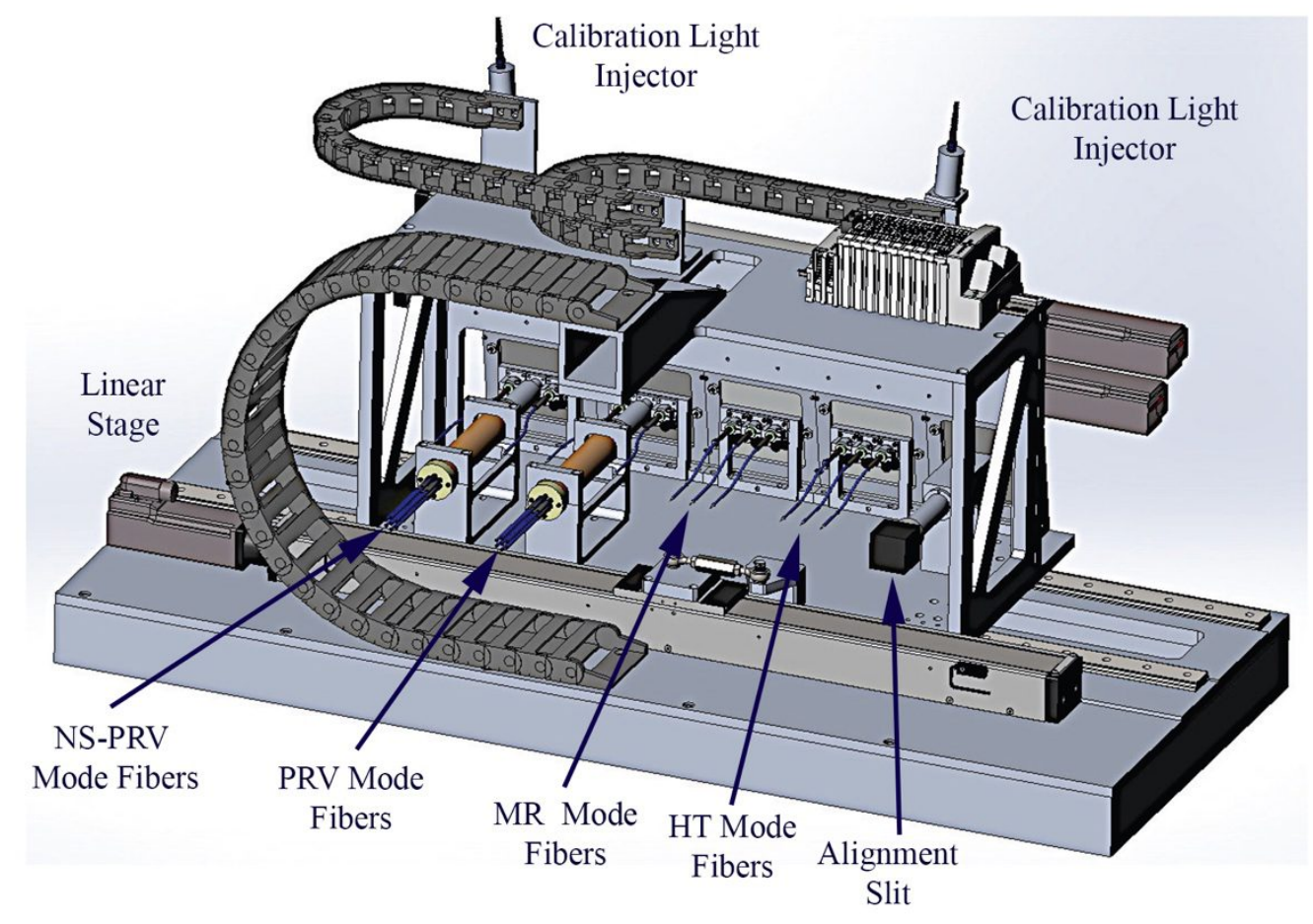

Figure 13: Rear view of the GCFEA science fiber interface.

The integration of the fibers to the slit mirrors is shown in Figure 14 for the case of the MR and HT modes. The focal ratio adjustment optics and fiber end is integrated into a ferrule that is then attached to the back of the slit mirror and registered 
with a precision bore. Each fiber has an independent blade shutter that is actuated with a small bi-stable solenoid manufactured by Daco Instruments.
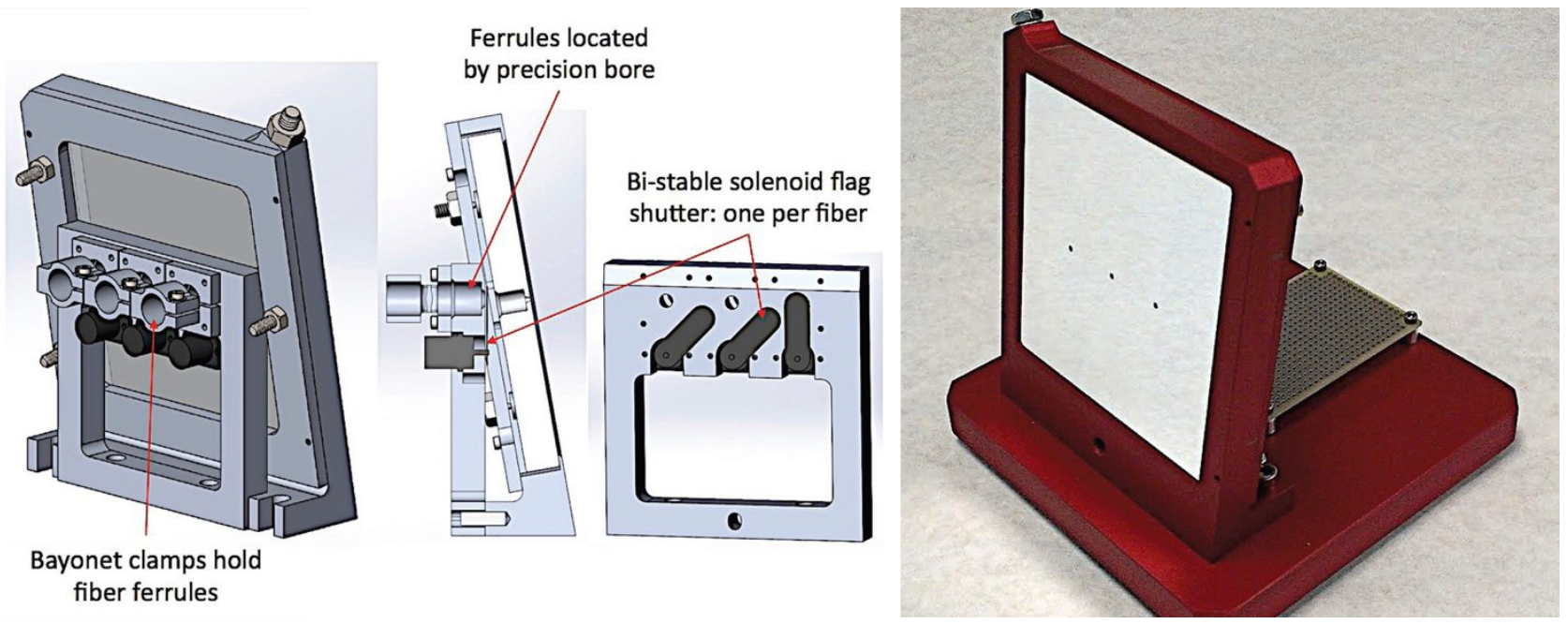

Figure 14: Slit mirror and science fiber interface. Left panel: Back view of slit mirror showing fiber interface of modes that are not pupil slices (MR \& HT modes). Center panel: Sectioned view of slit mirror showing fiber interface and detail of individual fiber shutters. Right panel: Slit mirror prototype, front view.

The treatment of fibers at the spectrograph end of the GCSFIB is shown in Figure 15 and Figure 16. The fibers that constitute each observing mode are broken out independently, as are the MANIFEST fibers (not shown). Because of the close proximity of the fiber slit and the Mangin mirror, they are mounted as a single unit.

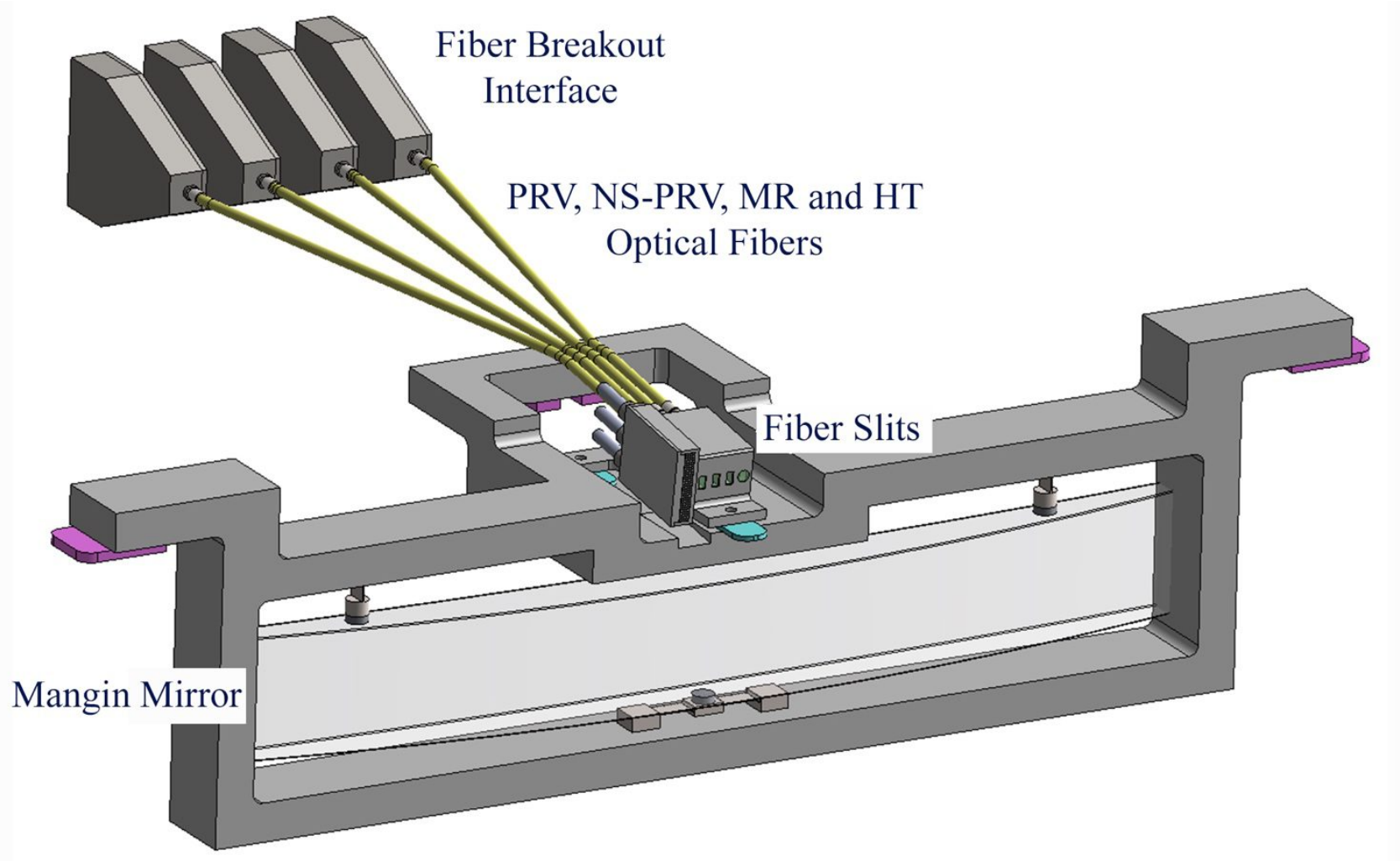

Figure 15: Mounting of the G-CLEF fiber slit. The fiber slit integrated into the Mangin mirror mount. 
The focal ratio adjustment optics of the GCSFIB and MANIFEST are intrinsically different. The GCSFIB optics produce a real image of the science fibers somewhat forward of the optics. The MANIFEST optics will produce a virtual pupil image that constitutes the entrance to the spectrograph. For this reason, the GCSFIB and MANIFEST fibers are pistoned with respect to each other (Figure 16).

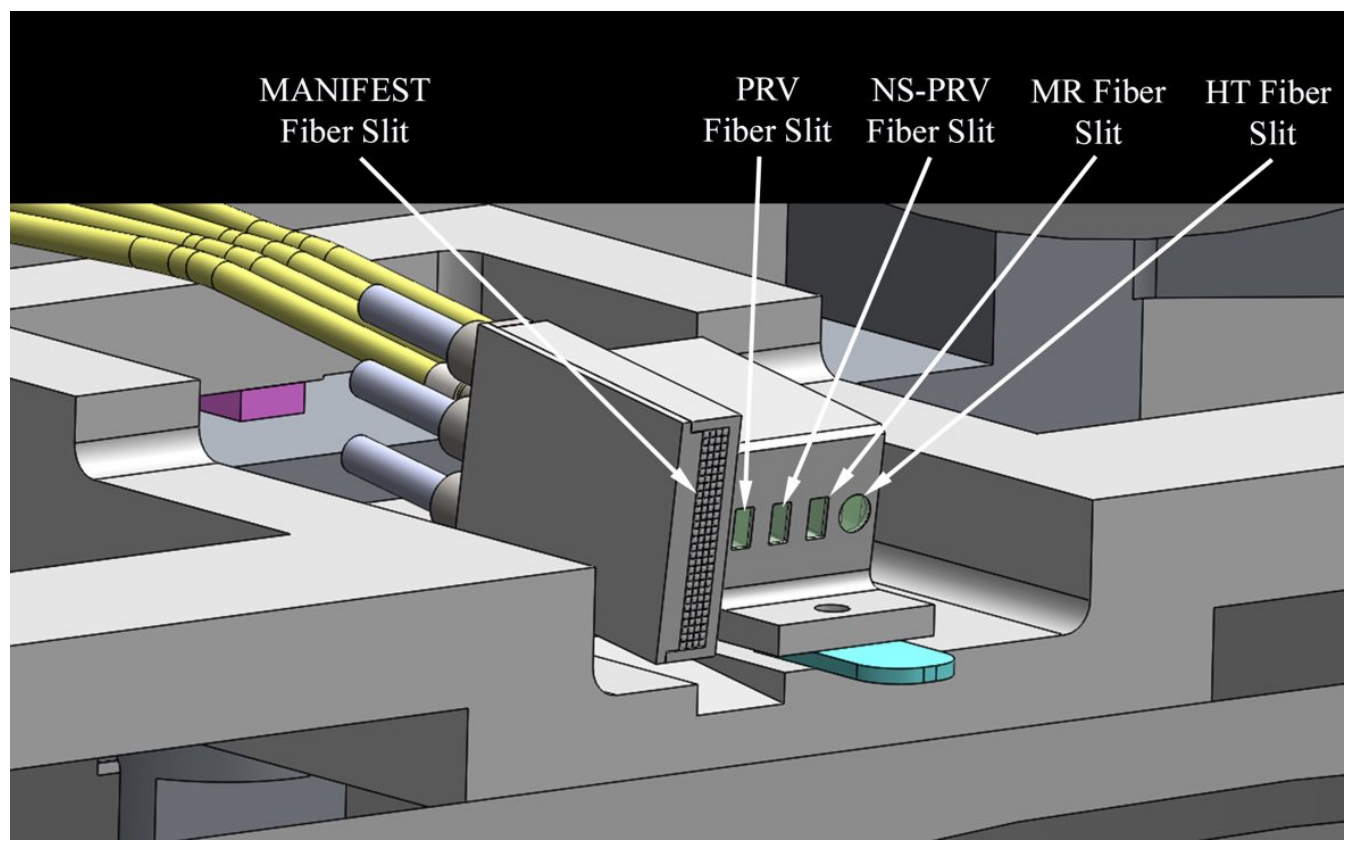

Figure 16: Detail of G-CLEF spectrograph fiber slit showing location of slits for all G-CLEF modes.

\subsection{GCFEA Mechanical Design}

The mechanical design of the GCFEA is shown in Figure 17. There are two major subsystems that constitute the GCFEA. The FCC, fiber interface, front end shutter and the rear beam relay triplet lens are mounted on the GMT instrument platform. The tertiary, ADC, and half the relay system are mounted on the Gregorian Instrument Rotator (GIR). This subsystem can be retracted so the telescope can operate in Direct Gregorian mode or a second tertiary can be inserted to fold the telescope beam to other GMT focal plane instruments. The GMT mount is an altitude-azimuth design, so in normal operations for other instruments, the GIR rotates to compensate field rotation. For G-CLEF observations, the GIR is not rotated. The split design was adopted for a number of reasons, most prominently that the optical fibers could not be mounted on the rotating GIR.

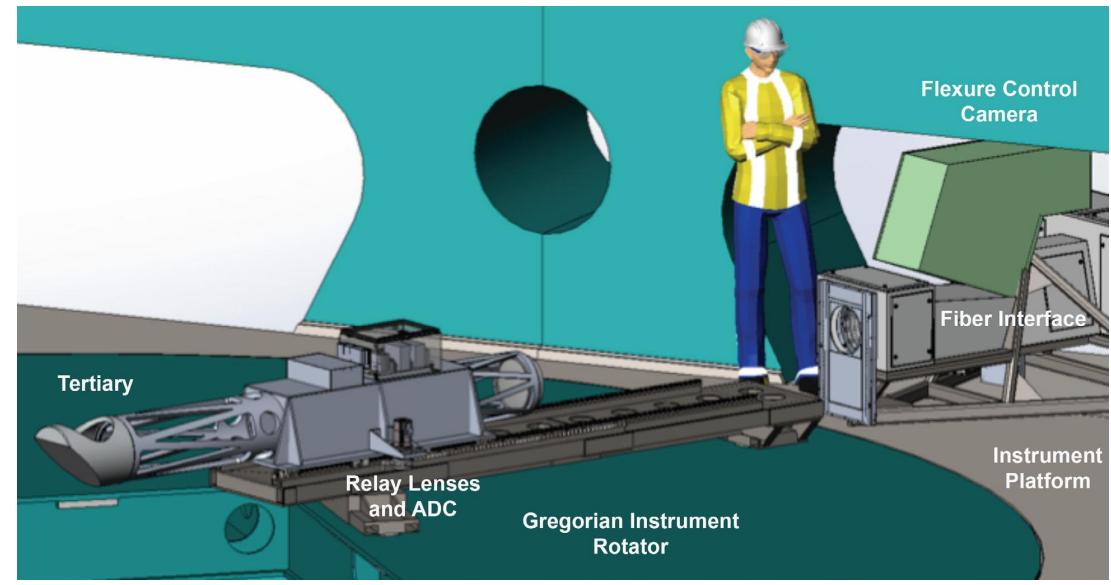

Figure 17: Mechanical layout of the GCFEA components. The deployable module at the left is mounted on the telescope Gregorian Instrument Rotator (GIR). The components on the left - the FCC and fiber interface - are mounted on the fixed instrument platform. 
After PDR, the mechanical design of the FCC, shown in Figure 18, underwent revision to accommodate slight changes in telescope architecture and simplify the intrinsic design. The internal pupil of the FCC is instrumented with two filter wheels to allow insertion of a selection of band-pass filters or the tent prism for focus adjustment. At PDR, the focal plane was static and derotation was implemented by image post-processing. The FFC camera is now physically compensates field rotation by rotating the camera with a direct drive ("DD") motor.

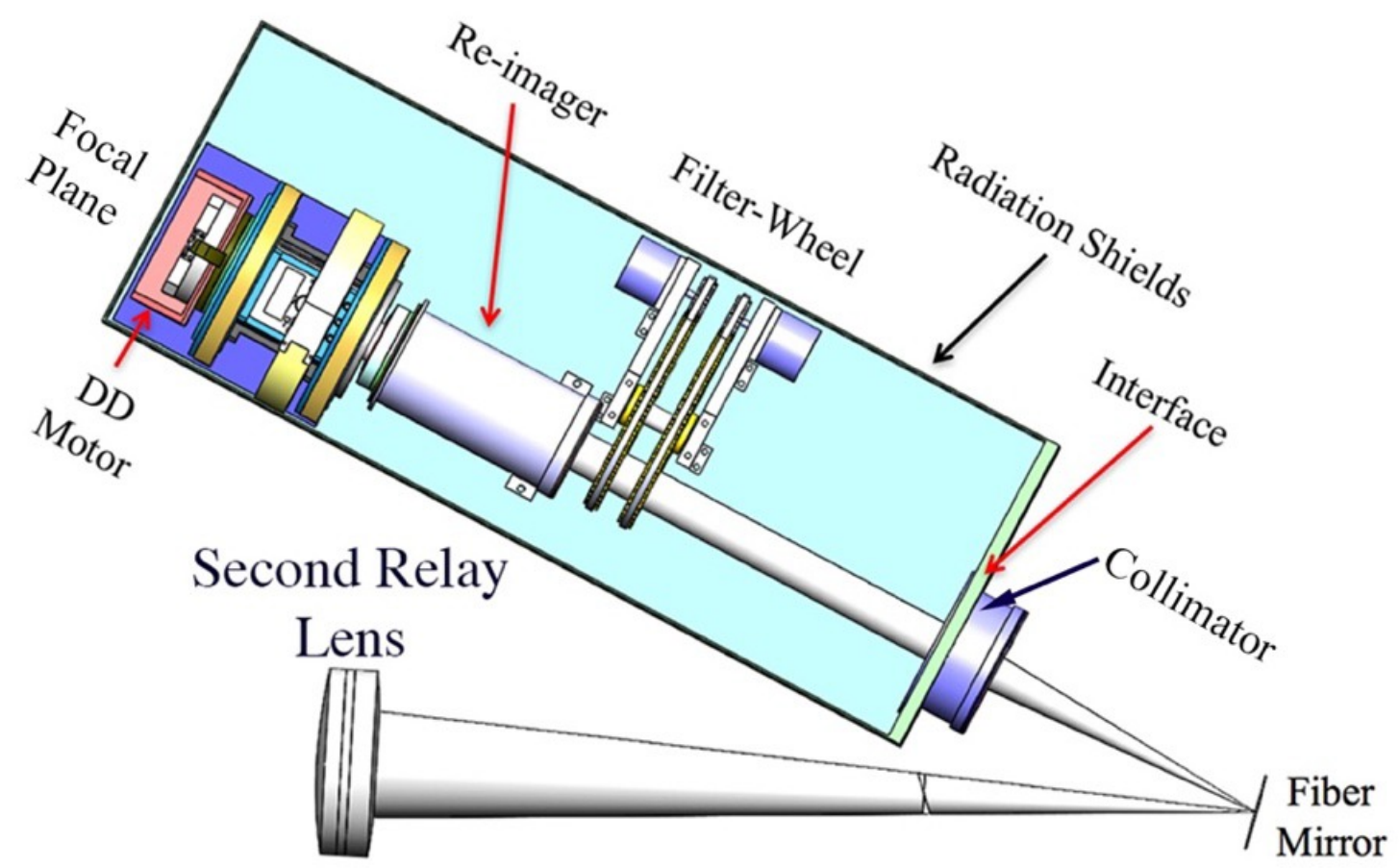

Figure 18: The G-CLEF FCC mechanical design.

\subsection{Calibration Lamp System Mechanical Design}

As was discussed earlier, the current lack of metallic cathode thorium argon lamps and the rapid advances in alternate calibration protocols for PRV science make it likely that the actual suite of calibrators we will use with G-CLEF will be modified between this writing and first light. However, all of the new calibrators are too large to integrate into the calibration lamp box, so they will be mounted externally and feed integrating spheres within the calibration lamp box. However, there is a deep experience base performing wavelength calibrations using thorium argon hollow cathode lamps for wavelength calibration. For this reason, it seems likely that thorium argon hollow cathode lamps will be included in the calibration system for science programs that do not need ultimate RV precision.

With the caveat that the makeup of the G-CLEF calibrators will change in the future, the baseline calibration lamp system is shown in Figure 19. Based on the HARPS model of calibration, there are three thorium argon lamps - i) a high current lamp which has a relatively short lifetime for short, frequent calibrations, ii) a low current lamp which lasts considerably longer and is used for reference calibrations, and iii) a spare. The baseline tungsten halogen lamp shown in the figure will probably be replaced with an integrating sphere fed with a laser-driven light source for continuum calibration. The integrating sphere shown in Figure 19 will probably be fed with a LFC or etalon. 


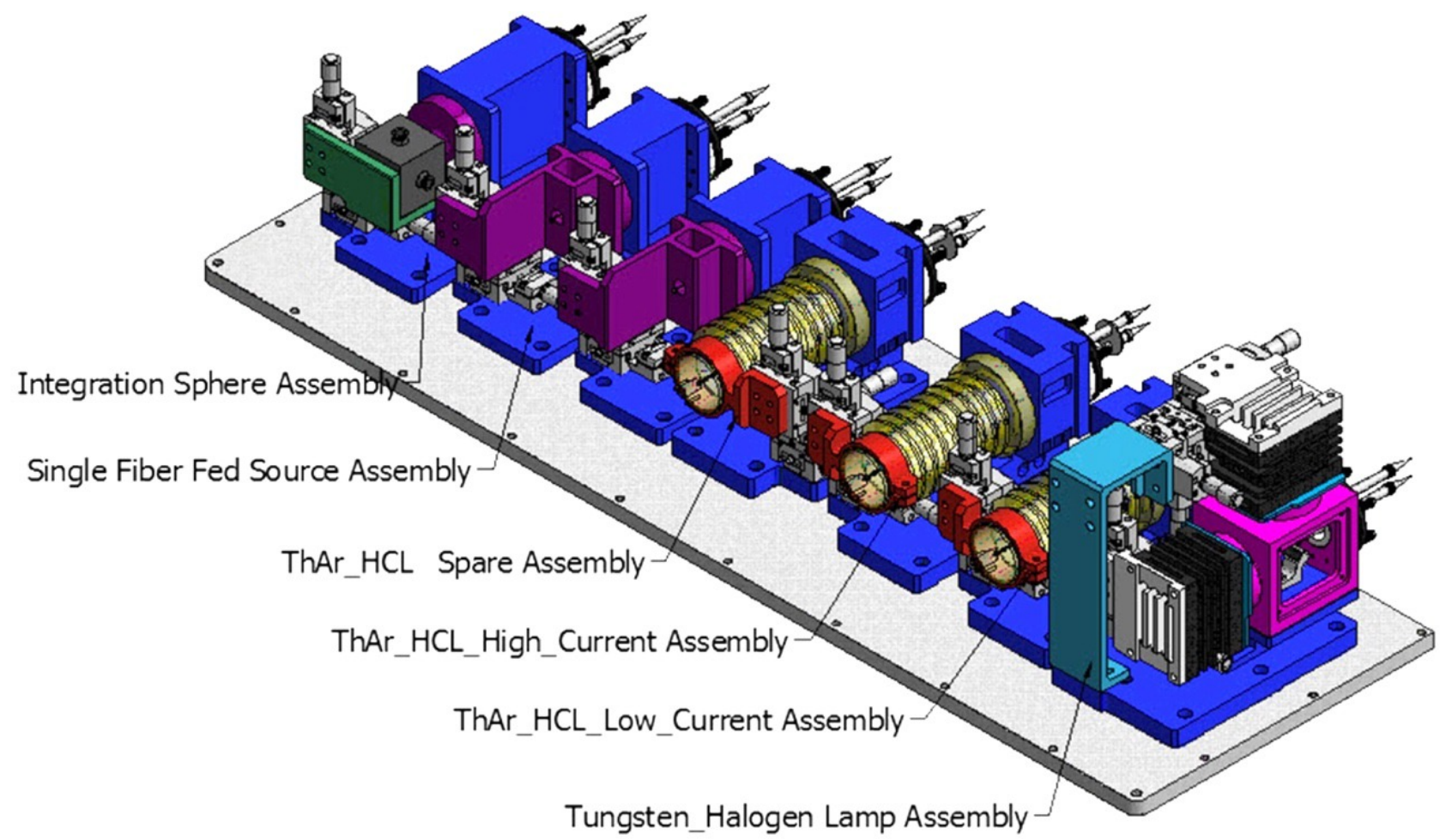

Figure 19: Calibration lamp light sources at PDR.

The light source selection system is shown in Figure 20. The fiber carriages that hold the GCCFIB ends and the injection lenses ride on ball screws which are guided by the linear motion guide. The ball screws are actuated with stepper motors and the GCCFIB fibers are protected in energy chains.

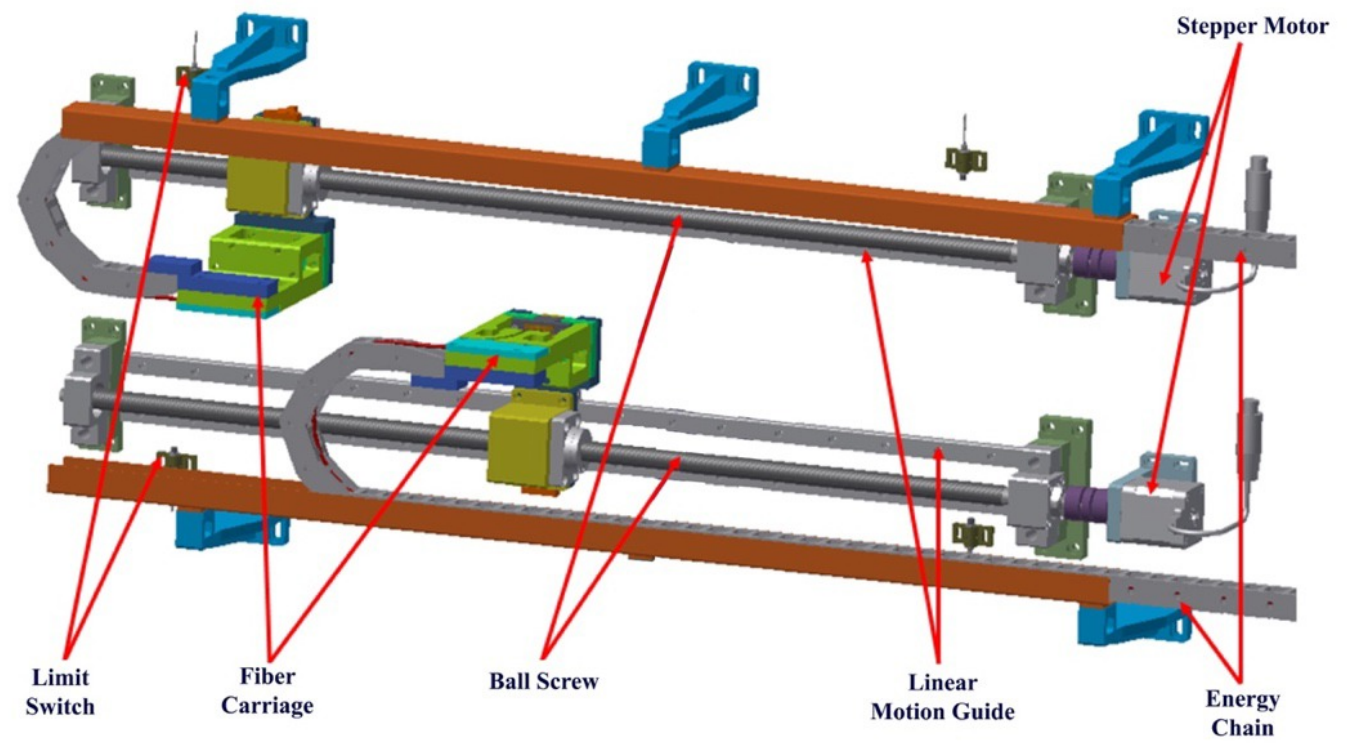

Figure 20: Calibration lamps system light source selection system. 
Each of the lamp housing types have a unique design. For illustrative purposes we show the thorium argon hollow cathode lamp housing in Figure 21. In addition to supplying power and heat-sinking the lamp, the position of the lamp is adjusted with micrometers in the $\mathrm{X}, \mathrm{Y}$ and $\mathrm{Z}$ direction. A photodiode monitors light scattered by the lamp envelop to verify that the lamp is operating and measures the intensity of the light produced by the lamp. All light sources share these features.
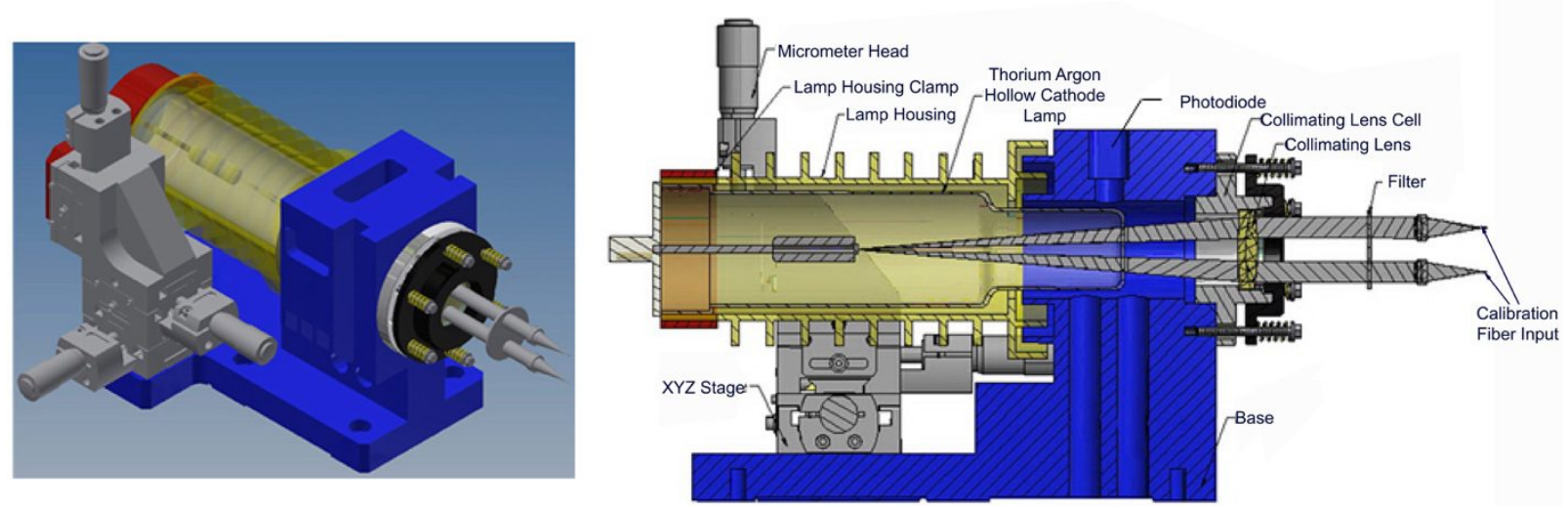

Figure 21: Thorium-argon hollow cathode lamp housing.

\section{SCHEDULE}

G-CLEF in currently several months into critical design phase. Critical design review is planned for May 2017. AIT at the GMT is scheduled to start in mid-2021 and first light is planned for early 2022.

\section{REFERENCES}

[1] Szentgyorgyi, A., et al., "A preliminary design for the GMT-Consortium Large Earth Finder (G-CLEF)," Proc. SPIE 9147, 914726 (2014).

[2] Szentgyorgyi, A., et al., "The GMT-CfA, Carnegie, Catolica, Chicago Large Earth Finder (G-CLEF): a general purpose optical echelle spectrograph for the GMT with precision radial velocity capability," Proc. SPIE $8446,84461 \mathrm{H}$ (2012).

[3] Ricker, G., et al, “Transiting Exoplanet Survey Satellite (TESS),” JATIS, 1, 014003 (2015).

[4] Fortier, A., Beck, T., Benz, W., Broeg, C., Cessa, V., Ehrenreich, D. and Thomas, N., "CHEOPS: a space telescope for ultra-high precision photometry of exoplanet transits," Proc. SPIE 9143, 91432J (2014).

[5] Mégevand, D., et al. "ESPRESSO: the radial velocity machine for the VLT," Proc. SPIE 9147, 91471H (2014).

[6] Quirrenbach, A., et al.,"CARMENES instrument overview," Proc SPIE 9147, 91471F (2014).

[7] Schwab, C., et al.,"'Optical design for NEID, a proposed spectrometer for NASA's WIYN extreme precision Doppler spectrometer," Proc. SPIE 9908, 9908-282 (2016).

[8] Gibson, S. et al., "SHREK: Stable High-Resolution Echelle for Keck"” Proc. SPIE, 9908-265 (2016).

[9] Jurgenson, C., Fischer, D., McCracken, T., Sawyer, D., Szymkowiak, Muller, G. and Santoro, F.,"EXPRES: a next generation RV spectrograph in the search for earth-like worlds", Proc. SPIE 9908, 9908-258 (2016).

[10] Seifahrt, A, Strümer and Bean, J, "A micro-lens array based pupil slicer and double scrambler for MAROON-X", Proc. SPIE 9912, 9912-61 (2016).

[11] Rodler, F. and Lopez-Morales, M., "Feasibility Studies for the Detection of O2 in an Earth-like Exoplanet", ApJ, 781, 54 (2014).

[12] Snellen, I., de Kok, R., le Poole, R., Brogi, M and Birkby, J., “ Finding Extraterrestrial Life Using Ground-based High-dispersion Spectroscopy,", ApJ, 764, 182 (2013).

[13] Saunders, W., Colless, M. Saunders, I., Hopkins, A., Goodwin, M., Heijmans, J., Brzeski, J. and Farrell, T., "MANIFEST: a multi-instrument fiber positioning system for GMT", Proc. SPIE 7735, 773568 (2010). 
[14]Ben-Ami, S., Epps, H., Podgorski, W. and Szentgyorgyi, A.," The optical design of the G-CLEF Spectrograph: the first light instrument for the GMT,", Proc. SPIE. 9908, 9908-374 (2016).

[15]Furesz, G., et al.,"The G-CLEF spectrograph optical design”, Proc. SPIE 9147, 91479G (2014).

[16] Mueller, M. Szentgyorgyi, A., Baldwin, D., Ben-Ami, S., Evans, I., Foster, J., Gauron, T.M., McMuldroch, S., Plummer, D., and Podgorski, W., "The opto-mechanical design of the GMT-Consortium Large Earth Finder (GCLEF)", Proc. SPIE 9908, 9908-375 (2016).

[17] Evans, I., et al., " Software requiremennts flowdown and preliminary software design of the G-CLEF spectrograph,", Proc. SPIE. 9913, 9913-62 (2016).

[18] Podgorski, W. at al., "A novel systems engineering approach to the design of a precision radial velocity spectrograph: the GMT-Consortium Large Earth Finder (G-CLEF)," Proc. SPIE, 9147, 91478W (2014).

[19] Jacoby, G., Bernstein, R., Bouchez, A., Colless, M., DePoy, D., Espeland, B., Jaffe, D., Lawrence, J., Marshall, J., McGregor, P. Sharp, R., Szentgyorgyi, A. and Walls, B., "Instrumentation Progress at the Giant Magellan Telescope project," Proc. SPIE 9908, 9908-68 (2016).

[20] Baranne, A., "White Pupil Story or Evolution of a Spectrographic Mounting", ESO Conference and Workshop Proceedings, 1195-1206, (1988).

[21] McLeod, B., et al., "The Giant Magellan Telescope active optics system”, Proc. SPIE 9145, 91451T (2014).

[22] Oh, J.-S., et al.,"Opto-mechanical design of the G-CLEF flexure control camera system," Proc. SPIE 9908, 9908-356 (2016).

[23] McCarthy, P., Fanson, J. and Bernstein, R., "Overview and status of the Giant Magellan Telescope Project", Proc. SPIE 9906, 9906-38 (2016).

[24] Burgh, E., Bershady, M., Westfall, K. and Nordsieck, K., "Recombination Ghosts in Littrow Configuration: Implications for Spectrographs Using Volume Phase Holographic Gratings:, PASP, 859, 1069-1082 (2007).

[25] Phillips, D., et al., "An astro-comb calibrated solar telescope to search for the radial velocity signature of Venus", Proc. SPIE 9912, 9912-243 (2016)

[26] Murphy, M., Locke, C., Light, P., Luiten, A and Lawrence, J.,"Laser frequency comb technique for precise astronomical spectroscopy", MNRAS, 422,761-771 (2012)

[27] Wildi, F., Pepe, F., Chazelas, B., Lo Curto, G. and Lovis, C.,"'A Fabry-Perot calibrator for the HARPS radial velocity spectrograph, performance report", Proc. SPIE7735, 7735E (2010).

[28] McCracken, T., Jurgenson, C., Fischer, D., Stoll, R., Szymkowiak, A., Bradford, J. and Rutter, W.,"Single lock: a stable Fabry-Perot based wavelength calibrator", Proc. SPIE 9147, 9147E (2014).

[29] Pepe, F., et al., "Performance verification of HARPS: first laboratory results", Proc SPIE 4841, 4841, 1045-1056 (2003).

[30] Baldwin, D., Podgorski, W., Mueller, M., McCracken, K., Bergner, H. and Foster, J., “Advanced structural design for precision radial velocity instruments", Proc SPIE 9912, 9912-121 (2016).

[31] Vogt, S., et al., "HIRES: the high-resolution echelle spectrometer on the Keck 10-m Telescope", Proc. SPIE, 2198, 362 (1994).

[32] Megevand, D. et al."ESPRESSO: the radial velocity machine for the VLT”, Proc. SPIE 9147, 91471H (2014).

[33] Lizon, J.-L., Iwet, O., Deiries, S. Hinterschuster and R., Dekker, J., "An ultra-stable cryostat for the detectors of ESPRESSO”, Proc.SPIE 9908, 9908-232 (2016). 